\title{
Exercise promotes a cardioprotective gene program in resident cardiac fibroblasts
}

\author{
Janet K. Lighthouse,, Ryan M. Burke, 'Lissette S. Velasquez, ${ }^{1}$ Ronald A. Dirkx Jr., ${ }^{1}$ \\ Alessandro Aiezza II, ${ }^{1}$ Christine S. Moravec, ${ }^{2}$ Jeffrey D. Alexis, ${ }^{3}$ Alex Rosenberg, ${ }^{4}$ \\ and Eric M. Small ${ }^{1,3,5}$ \\ ${ }^{1}$ Aab Cardiovascular Research Institute, University of Rochester School of Medicine and Dentistry, Rochester, New York, \\ USA. ${ }^{2}$ Department of Molecular Cardiology, Cleveland Clinic, Cleveland, Ohio, USA. ${ }^{3}$ Department of Medicine, ${ }^{4}$ Department \\ of Allergy, Immunology, and Rheumatology Research, and ${ }^{5}$ Department of Pharmacology and Physiology, University of \\ Rochester School of Medicine and Dentistry, Rochester, New York, USA.
}

Exercise and heart disease both induce cardiac remodeling, but only disease causes fibrosis and compromises heart function. The cardioprotective benefits of exercise have been attributed to changes in cardiomyocyte physiology, but the impact of exercise on cardiac fibroblasts (CFs) is unknown. Here, RNA-sequencing reveals rapid divergence of CF transcriptional programs during exercise and disease. Among the differentially expressed programs, NRF2-dependent antioxidant genes - including metallothioneins ( $\mathrm{Mt1}$ and $\mathrm{Mt2}$ ) - are induced in CFs during exercise and suppressed by TGF- $\beta / p 38$ signaling in disease. In vivo, mice lacking Mt1/2 exhibit signs of cardiac dysfunction in exercise, including cardiac fibrosis, vascular rarefaction, and functional decline. Mechanistically, exogenous MTs derived from fibroblasts are taken up by cultured cardiomyocytes, reducing oxidative damage-dependent cell death. Importantly, suppression of MT expression is conserved in human heart failure. Taken together, this study defines the acute transcriptional response of CFs to exercise and disease and reveals a cardioprotective mechanism that is lost in disease.

Conflict of interest: EMS is the recipient of a research grant from Novartis Pharmaceuticals and is a co-inventor on a US patent $(9,937,156)$.

License: Copyright 2019, American Society for Clinical Investigation.

Submitted: December 7, 2016 Accepted: November 20, 2018 Published: January 10, 2019

\section{Reference information:} JCl Insight. 2019;4(1):e92098. https:// doi.org/10.1172/ji.insight.92098.

\section{Introduction}

The heart responds to excessive workload through compensatory hypertrophic growth. Hypertrophic growth caused by pressure overload or ischemic injury is accompanied by cardiomyocyte (CM) death and the formation of scar tissue, which can lead to heart failure (HF) and lethal arrhythmias (1). Although the heart also grows in size in response to the increased metabolic demands of exercise, physiological hypertrophy does not elicit a fibrotic response and remains an adaptive process (1-3). Accumulating evidence from clinical studies and animal models underscores the cardioprotective benefits of exercise via supporting CM function and viability (4-6). However, relatively little is known about how cardiac fibroblasts (CFs) respond to physiological stimuli and how they may contribute to cardiac health.

CFs are primarily studied in the context of pathological events, which lead to changes in mechanical tension and neurohumoral signals that stimulate their proliferation and activation (7-11). Activated CFs, called myofibroblasts, secrete extracellular matrix (ECM) in an adaptive response to injury that contributes to cardiac repair and prevents lethal wall rupture following myocardial infarction (MI) (12-16). However, sustained CF activation ultimately leads to excessive fibrosis and ventricle wall stiffening.

Recent studies reveal additional roles for CFs in normal cardiac homeostasis. CFs influence processes such as electrical conduction, inflammation, angiogenesis, and cardiac growth and development (17-22). CFs communicate with neighboring cells via junction proteins or secretion of growth hormones, cytokines, and inflammatory signals $(21,22)$. Defining the CF response to pathological and physiological cues is critical to understanding the cellular and molecular mechanisms that underlie the cardioprotective benefits of exercise that might be harnessed for therapeutic purposes.

In this study, we utilize RNA-sequencing (RNA-Seq) to investigate the gene expression changes occurring in CFs during physiological (swim training) and pathological (pressure overload or ischemia) cardiac remodeling. Pathological remodeling induced Rho/Rho-kinase and serum response factor-dependent (SRF-dependent) expression of myofibroblast gene programs (9, 14, 23-26). In contrast, physiological 
remodeling did not stimulate the expression of genes encoding ECM proteins and canonical myofibroblast markers. Unexpectedly, a program of NFE2L2-dependent (NRF2-dependent) ROS and toxin scavengers was suppressed in pathological CFs and elevated in exercise.

Among the NRF2-dependent genes are metallothioneins (Mt1 and Mt2), which are enriched in CFs compared with CMs and suppressed in mouse models of disease. MTs contain thiol groups that sequester hydroxyl radicals (27) and were reported to protect neurons from oxidative damage in a paracrine manner (28). Here, we demonstrate that MT overexpression only mildly inhibits myofibroblast activation, but exogenous MTs are taken up by CMs, providing protection from oxidative stress. In vivo, $M t 1 / 2-\mathrm{KO}$ (MTKO) mice display a decline in cardiac function during strenuous exercise, characterized by fibrosis and reduced vascularization. Importantly, suppression of MT1 expression is conserved in human HF, which we show is at least partially dependent upon TGF- $\beta 1 /$ p38 MAPK signaling. Taken together, our study highlights a dynamic program of gene regulation in CFs during physiological and pathological cardiac remodeling that may underlie the cardioprotective benefits of exercise.

\section{Results}

Fibrosis genes are inversely regulated in fibroblasts during exercise and disease. To define the gene expression changes in CFs that underlie the divergent functional outcomes of exercise and disease, we developed an RNASeq screen using WT C57BL/6 mice subjected to a swimming regimen to induce physiological remodeling, transverse aortic constriction (TAC) to induce pressure overload induced remodeling, or MI to induce ischemic injury (Figure 1A). We observed subtle hypertrophic cardiac growth beginning at day 10 of the swimming protocol, which became highly significant at 28 days in both male and female mice and was not associated with the development of fibrosis or expression of Colla1 (Figure 1B and Supplemental Figure $1, \mathrm{~A}-\mathrm{D})(2,3)$. In contrast, we observed significant fibrosis and reduced cardiac function associated with pathological remodeling (Supplemental Figure 1, B, C, and E). Hypertrophic growth and functional decline was not observed 3 days after TAC, first becoming evident 10 days after TAC. Quantitative PCR (qPCR) was performed on left ventricular $\mathrm{CMs}$ to evaluate molecular markers of cardiac remodeling, revealing that the $M y h 7 / 6$ ratio increased only after pathological stimuli and that $C e b p \beta$ levels decreased consistently in male and female animals only after exercise, confirming pathological and physiological remodeling, respectively (Supplemental Figure 1F). Based on these observations, we chose to evaluate the transcriptional changes occurring in CFs following 3 or 4 days of remodeling (prior to morphological changes) and 10 days of remodeling (coincident with the earliest morphological changes) (Figure 1A).

We isolated CMs and nonmyocytes by differential adherence to reduce the possibility of bias toward a particular population of fibroblasts based on currently available molecular markers. Of the adherent nonmyocytes, flow cytometry confirmed that approximately $77 \%$ of adherent cells were not of CD $31^{+}, \mathrm{CD} 45^{+}$, or PDGFR $\beta^{+}$ vascular or hematopoietic lineages, verifying enrichment of ventricular fibroblasts (Supplemental Figure 1G; $P$ $<0.0001$ ). Importantly, we did not observe alterations in the proportion of $\mathrm{CD} 31^{+}$or $\mathrm{CD} 45^{+}$cardiac cells isolated from animals subjected to exercise or TAC (Supplemental Figure $1 \mathrm{H}$ ). Identity of the nonmyocyte populations was initially evaluated by qPCR, revealing enrichment in Fsp1 and depletion in cardiac Tnnt2 genes (Supplemental Figure 1I). Subsequent analysis of RNA-Seq data for markers of CMs (Myh6), smooth muscle cells (Myh11), endothelial cells (Cdh5, Pecam), and hematopoietic cells (Ptprc/CD45) revealed variable levels of expression around background, confirming nonfibroblasts were not differentially captured after exercise or disease (Supplemental Figure 1, J-L). However, further evaluation of RNA-Seq data revealed significant alterations in the expression of inflammatory markers $I l 1 \beta$, Tnf, and Nos2 during exercise and TAC, consistent with recent reports that fibroblasts act as sentinels in maintaining tissue homeostasis (Supplemental Figure 1L) (29).

Preliminary studies confirmed TAC upregulated Postn and Colla1 and decreased Tcf21 expression (Figure 1C), consistent with a recent report that Postn and Tcf21 mark the activated and quiescent fibroblast state, respectively (30). Initial analysis of RNA-Seq data revealed that TAC increased the expression of ECM and myofibroblast marker genes (Figure 1, D and E). In contrast, swimming reduced several ECM genes (Figure 1D). For example, Colla1 expression in CFs was reduced in response to swimming in both male and female mice, reaching statistical significance in males after 28 days (Supplemental Figure 1D). Together, these results are the first to reveal that CFs actively respond to both pathological and physiological cues.

The CF GEP rapidly diverges in exercise and disease. We next evaluated the RNA-Seq data to define the global gene expression programs (GEP) that emerge with the earliest changes in CF phenotype and cardiac physiology. Global patterns of gene expression were evaluated by Principal Component Analysis (PCA) 
and Spearman correlation, confirming consistency between biological replicates (Figure 2, A and B). This analysis also revealed active GEP changes in CFs during physiological and pathological remodeling. CFs isolated from mice 5 days after MI also exhibited a GEP that was distinct from controls, TAC, and swim samples. Sham and sedentary controls were indistinguishable from each other and were, thus, treated as a single group in further analyses. These initial results revealed that CFs differentially respond to pathological and physiological cues in as few as 3 days by enacting dynamic transcriptional changes.

To identify Gene Ontology (GO) pathways and individual genes that are differentially expressed in CFs in exercise or disease, we performed Ingenuity Pathway Analysis (IPA) (https://www.qiagenbioinformatics. $\mathrm{com}$ /products/ingenuity-pathway-analysis/). We limited our analysis to genes that had an fragments per kilobase per million reads $(F P K M)>1$ in any condition and displayed a significant change in expression between either 10-day swim versus control or 10-day TAC versus control $(q<0.05)$. This generated a list of 2454 genes in swim versus control and 4051 genes in TAC versus control. The gene lists were reduced to 379 and 1976 genes, respectively, after removing genes that appeared in both lists and changed in the same direction (Supplemental Figure 2A). Consistent with myofibroblast activation, the most significantly enriched GO terms identified by IPA in pathological fibroblasts included stimulation of Rho-dependent contractile pathways and integrin-linked kinase (ILK) signaling (Figure 2C and Supplemental Figure 2, C-E). GO terms that were enriched in CFs upon exercise included detoxification and redox pathways, whereas ILK signaling was suppressed (Figure 2C and Supplemental Figure 2, B and C).

Transcription factor activity prediction analyses based upon gene expression changes revealed SRF was significantly inhibited in swim versus TAC (Figure 2D), consistent with previous studies that report Rho-dependent stimulation of SRF and myocardin related transcription factor (MRTF) activity promote fibroblast activation $(9,25,26,31)$. Motif analysis using HOMER 2.0 independently identified SRF binding elements, or CArG motifs, as the most enriched transcription factor binding site within $5 \mathrm{~kb}$ of genes that are upregulated in 10-day TAC versus sham (Supplemental Figure 3). Additional transcriptional pathways dysregulated in pathological fibroblasts include P53 signaling, PPAR- $\gamma$ signaling, and KLF2-dependent transcription. Transcription factors that were significantly upregulated after swim compared with disease included a number of proinflammatory transcription factors such as STAT1, NFATC2, NFкB1 $\alpha$, and IRF3/5/7 (Figure 2D) (29). Consistent with the enrichment of detoxification pathways after exercise, transcription factor analysis also revealed activation of aryl hydrocarbon receptor (AHR) and NFE2L2-dependent (NRF2-dependent) transcriptional programs. Indeed, expression of the $N f e 2 l 2$ gene was enriched in exercise (Figure 2D). These data indicate that disease drives the Rho$\mathrm{SRF}$-dependent myofibroblast program and exercise promotes NRF2-dependent detoxification program.

Exercise induces a fibroblast ROS-scavenger pathway. To identify individual genes that contribute to the physiological versus pathological GEP in CFs, we generated a rank-ordered list of gene changes in swim or TAC versus control. Of the 2075 genes changed in swim or TAC versus controls, 2052 displayed concordant dysregulation and 23 genes displayed significant $(q<0.05)$ and discordant dysregulation in swim or TAC versus controls (Figure 3). Among the inversely regulated genes, known myofibroblast markers and ECM genes (red lines, Figure 3) such as Postn and Col3a1 are robustly enriched in TAC and reduced in swim training. Postn and Col3a1 segregated with Acta2 in cluster 8, which contains genes elevated in TAC and reduced in swim, compared with controls (Figure 4 and Supplemental Table 1).

Of the 23 inversely regulated genes, 14 were enriched during exercise and suppressed in TAC, and 7 of these encoded antioxidant proteins in the GST or MT families (blue lines, Figure 3). These antioxidant response genes segregated into clusters 3 and 12 (with Tcf21), which contain the majority of genes that are enriched in swim compared with control and suppressed in TAC (Figure 4 and Supplemental Table 1).

The GST family (composed of GSTA, GSTM, GSTO, GSTP, GSTT, and GSTZ subfamilies) binds to reduced glutathione (GSH) to removes ROS and other compounds (32). While depletion of the cytosolic pool of GSH is highly correlated with disease, GST mutations have also been associated with disease, presumably by affecting clearance of GSH (33). We observed that various genes encoding GST family members displayed high FPKM values in swim and low FPKM values in TAC, which was confirmed by qPCR (Figure 5, A and B).

Metallothioneins are a family of small, cysteine-rich proteins that sequester heavy metals and act as free radical scavengers. Our RNA-Seq also revealed that $M t 1$ and $M t 2$ were enriched in swim compared with control CFs and were suppressed in TAC (Figure 5C). We confirmed by qPCR that $M t 1 / 2$ are maintained in CFs after physiological stimuli and lost in CFs after pathological stimuli; $M t 1 / 2$ are also much more highly expressed in CFs compared with CMs in all conditions and are not detected in CMs following TAC (Figure 5D). 
A

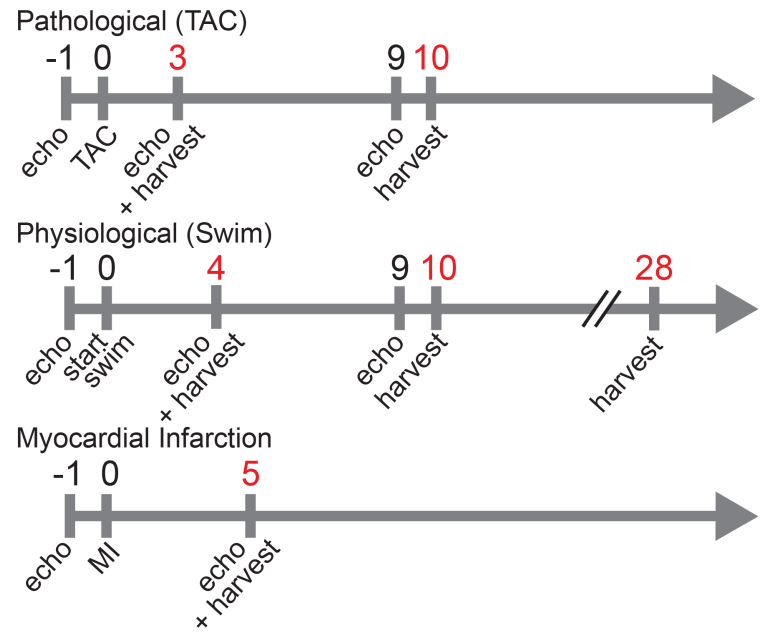

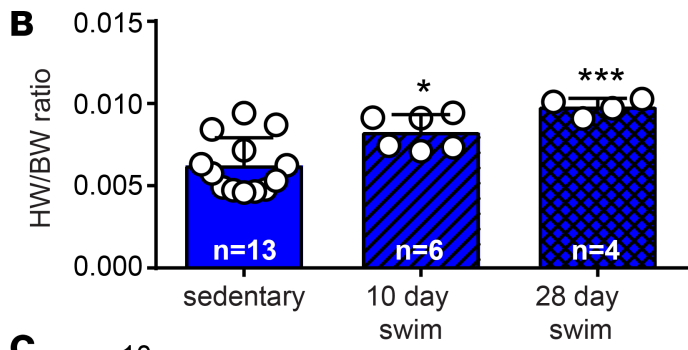
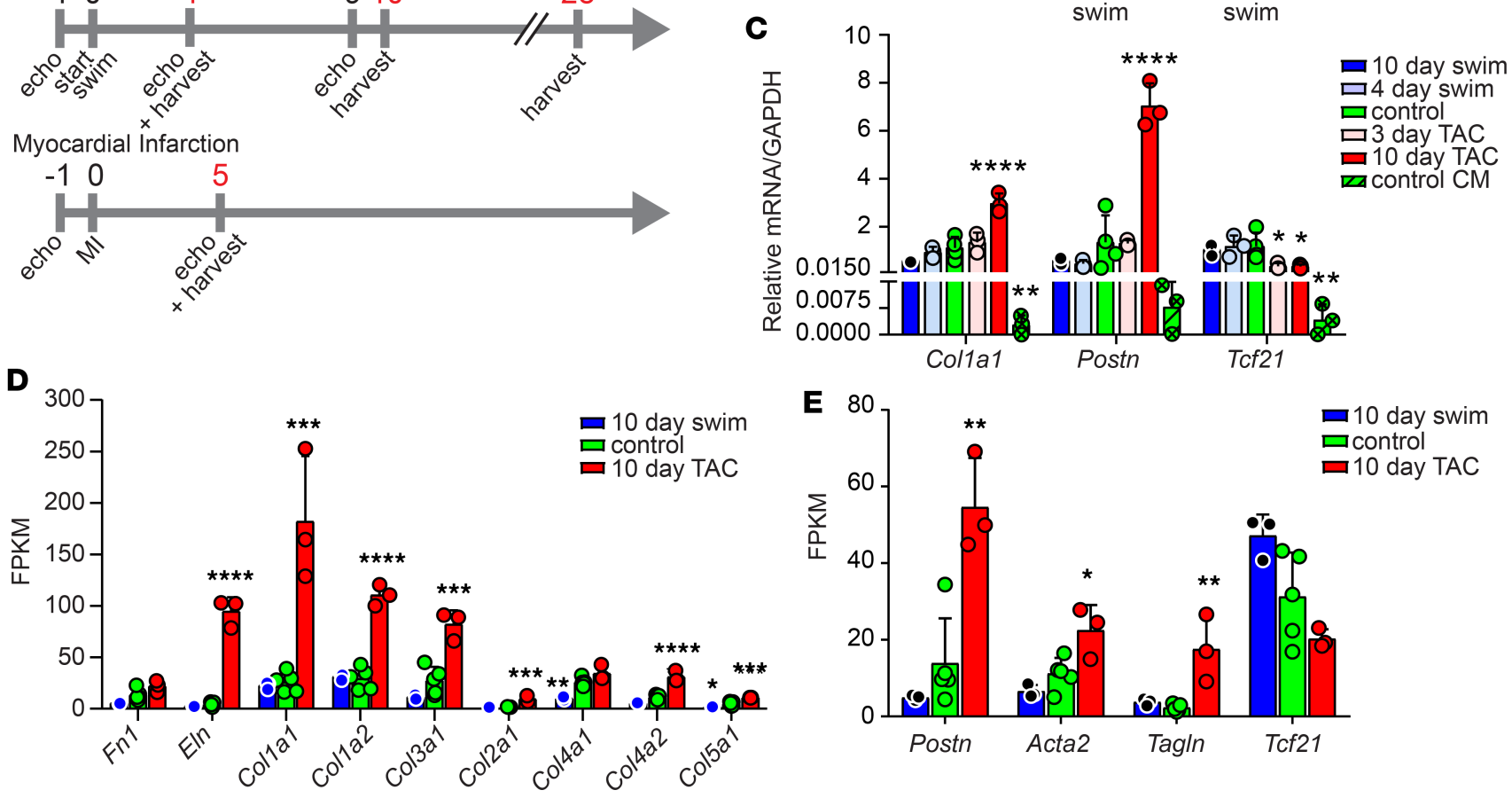

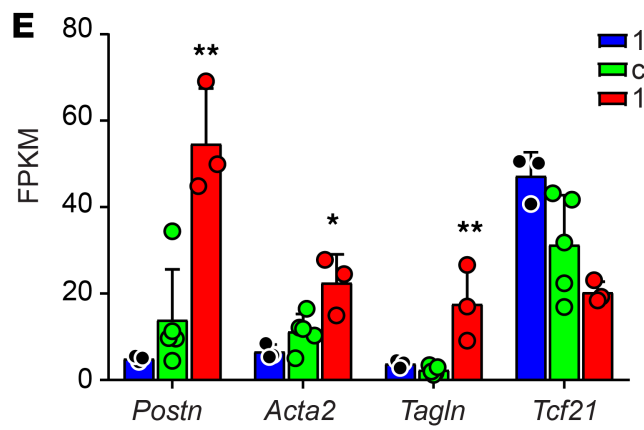

Figure 1. Evaluating the fibroblast response to physiological and pathological cardiac remodeling. (A) Experimental timeline (days) of inducing and assessing remodeling and isolating CFs (red) for RNA-Seq and subsequent analysis in C57BL/6 animals. (B) Heart weight/body weight (HW/ BW) ratio of sedentary and swim-trained animals. (C) qPCR analysis of primary CFs from mice subjected to the indicated treatment. (D) Analysis of fragments per kilobase of transcript per million mapped reads (FPKM) from RNA-Seq data for indicated ECM genes. (E) Analysis of FPKM from RNA-Seq data for CF and myofibroblast markers. Statistics in B-E were performed using 1-way ANOVA and Tukey's post-hoc test ( $n=3$ except for control where $n=5$ ). ${ }^{*} P<0.05,{ }^{* *} P<0.01,{ }^{* *} P<0.001,{ }^{* * *} P<0.0001$.

Inverse regulation of $M t 1 / 2$ expression in CFs by IGF-1 and TGF- $\beta 1 / p 38 M A P K$. The promoter regions of the $M t 1 / 2$ genes, along with several Gst genes, contain antioxidant response elements (AREs) that bind NRF2 and confer transcriptional responsiveness to ROS (34). Since Nfe2l2 gene expression and NRF2-dependent gene programs are increased in fibroblasts after 10 days of swimming and suppressed in disease, we evaluated NRF2 protein levels in CFs subjected to in vitro models of physiological or pathological remodeling. Primary CFs were cultured with $\mathrm{H}_{2} \mathrm{O}_{2}$ (ROS) to induce the NRF2-dependent antioxidant gene program (35), or ROS and insulin-like growth factor-1 (IGF-1) to model physiological cues that stimulate Akt phosphorylation and cardiac remodeling in exercise (Supplemental Figure 4, A and B) (5, 36). ROS and IGF-1/ ROS treatment of neonatal mouse CFs led to accumulation of NRF2 in the nucleus (Figure 6, A and B) and reduced ubiquitination of NRF2 (Supplemental Figure 4, C and D). Consistent with changes in NRF2 levels and localization, ROS or IGF-1/ROS treatment increased expression of Mt1/2 (Figure 6C). CFs were also cultured in the presence of TGF- $\beta 1 /$ ROS to model Smad2/3-dependent pathological conditions (Supplemental Figure 4, A and B). In contrast to IGF-1/ROS treatment, TGF- $\beta 1 /$ ROS increased cytoplasmic NRF2 ubiquitination and prevented nuclear accumulation of NRF2 (Figure 6, A and B, and Supplemental Figure 4, C and D). Furthermore, TGF- $\beta 1 /$ ROS treatment increased Acta2 and Colla1 expression and significantly attenuated $M t 1 / 2$ expression (Figure 6C). Thus, IGF-1 and TGF- $\beta 1$ modulate the impact of ROS on NRF2 levels and the ROS scavenger gene program in CFs.

TGF- $\beta$ signaling can drive pathological hypertrophy through canonical Smad signaling or noncanonical p38 MAPK-dependent signaling. p38 activity is disrupted in the heart upon pressure overload (37), and upstream 
A

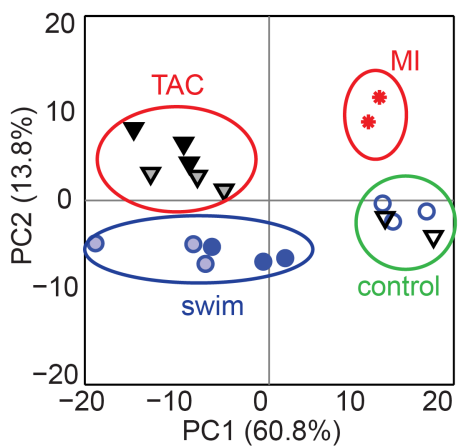

C

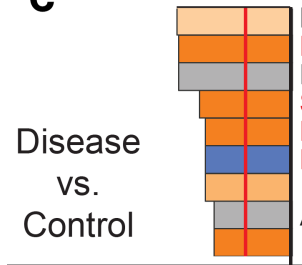

Death Receptor Signaling Regulation of Actin-based Motility by Rho Hepatic Fibrosis/Hepatic Stellate Cell Activation Signaling by Rho Family GTPases RhoA Signaling

RhoGDI Signaling

Induction of Apoptosis by HIV1

Agranulocyte Adhesion and Diapedesis ILK Signaling

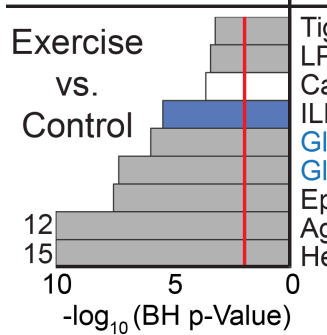

Tight Junction Signaling

LPS/IL-1 Mediated Inhibition of RXR Function Calcium Signaling

ILK Signaling

Glutathione Redox Reactions I

Glutathione-mediated Detoxification

Epithelial Adherens Junction Signaling

Agranulocyte Adhesion and Diapedesis Hepatic Fibrosis/Hepatic Stellate Cell Activation 0

$-\log _{10}(\mathrm{BH}$ p-Value $)$
B

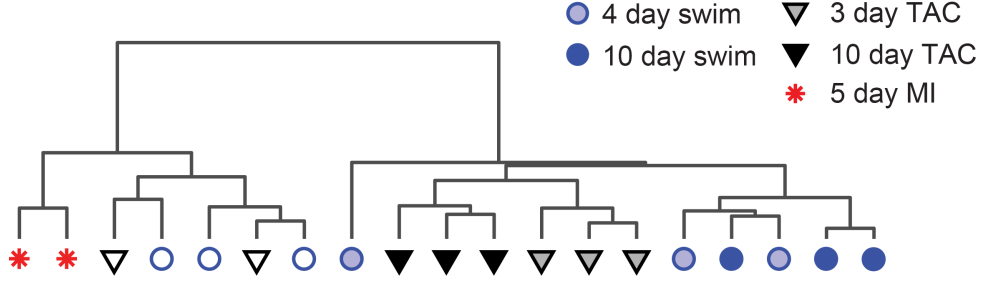

D

Exercise vs. Disease

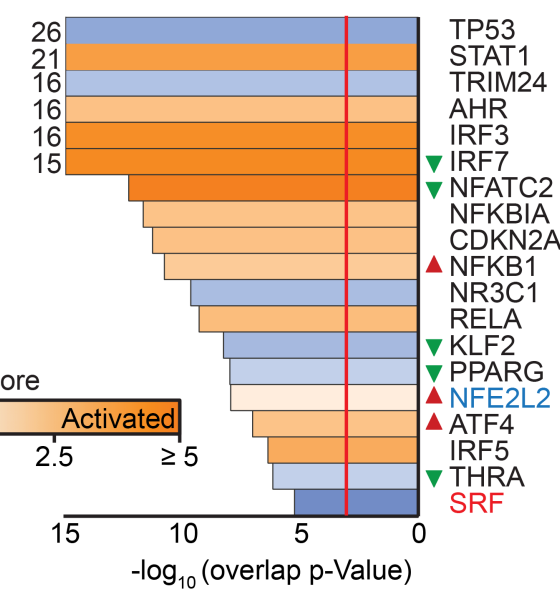

Figure 2. Gene expression profiling of pathological and physiological CFs. (A) PCA of log-transformed FPKM data from 19 individual CF samples from C57BL/ 6 animals of the indicated treatment group using the first and second principal components (PCs). There were 8500 of 23,359 genes included, where at least 5 of the 19 samples had a FPKM $>1$. No other feature selection was performed. (B) Spearman correlation and average linkage of the same 19 samples in $\mathbf{A}$ indicates consistency between biological replicates, which cluster in a hierarchical manner. (C) Ingenuity Pathway Analysis (IPA) of CFs at 10-day TAC (disease) versus control (top) or 10-day swim (exercise) versus control (bottom). The gray bars indicate that there is no activity pattern available identified in IPA, despite highly significant association of the genes within the pathway. Orange, positive z-score; white, zero z-score; blue, negative $z$-score; gray, no pattern. Vertical red line indicates a Benjamini-Hochberg-corrected $P=0.01$. (D) Predicted upstream regulators from 10-day swim versus 10-day TAC are shown that have overlap $P<0.001$ (red line). Red and green triangles indicate direction of change in expression in exercise versus disease, if significant. Red and blue text in $\mathbf{C}$ and $\mathbf{D}$ indicate myofibroblast regulation and detoxification pathways, respectively, and indicate a mechanistic link between TF and IPA canonical pathway.

regulators of p38 such as MKK3/6 stimulate the myofibroblast phenotype $(38,39)$. However, the fibroblast-specific role of p38 in physiological versus pathological cardiac remodeling remains unclear. We therefore evaluated the phosphorylation state of $\mathrm{p} 38$ in CFs isolated from animals subjected to 10 days of swimming, TAC, or sedentary control. We observed that p38 phosphorylation in CFs was dysregulated in both pathological and physiological remodeling, compared with controls (Supplemental Figure 4E), which led us to investigate whether chemical inhibitors of the p38 pathway might impact $M t$ expression in pathological CFs. Indeed, a pan-p38 inhibitor (BIRB796) restored Mt1/2 gene expression in TGF- $\beta 1 /$ ROS-treated neonatal CFs (Figure 6D), revealing that TGF- $\beta / \mathrm{p} 38$ MAPK signaling is partially responsible for the suppression of $M t 1 / 2$ in disease.

Paracrine role of $M T$ in protecting CM from oxidative damage. To address the potential effect of MTs in cardiac cell physiology, we first assessed whether overexpression of $M t 1$ was capable of blunting the response of NIH-3T3 cells to TGF- $\beta 1$. Transfection with FLAG-MT1 induced specific overexpression of Mt1 and not Mt2 compared with control transfected cells and partially blocked TGF- $\beta 1$-dependent stimulation of Postn (Figure 7, A-C). However, expression of other myofibroblast markers such as Colla1 or Acta2 were modestly, but not significantly, reduced (Figure 7, D and E). Because we demonstrated that CFs produce significantly more $M t 1 / 2$ than CMs, we next hypothesized that MTs secreted from CFs might protect adjacent $\mathrm{CMs}$ from oxidative stress in a manner reminiscent of neuroprotective mechanisms $(28,40)$. To investigate the paracrine effect of MTs in the heart, we conducted conditioned media experiments by culturing neonatal mouse CMs with media isolated from NIH-3T3 cells expressing FLAG-MT1. Confocal microscopy revealed that fibroblast-produced FLAG-MT1 was internalized by CMs (Figure 7F). 


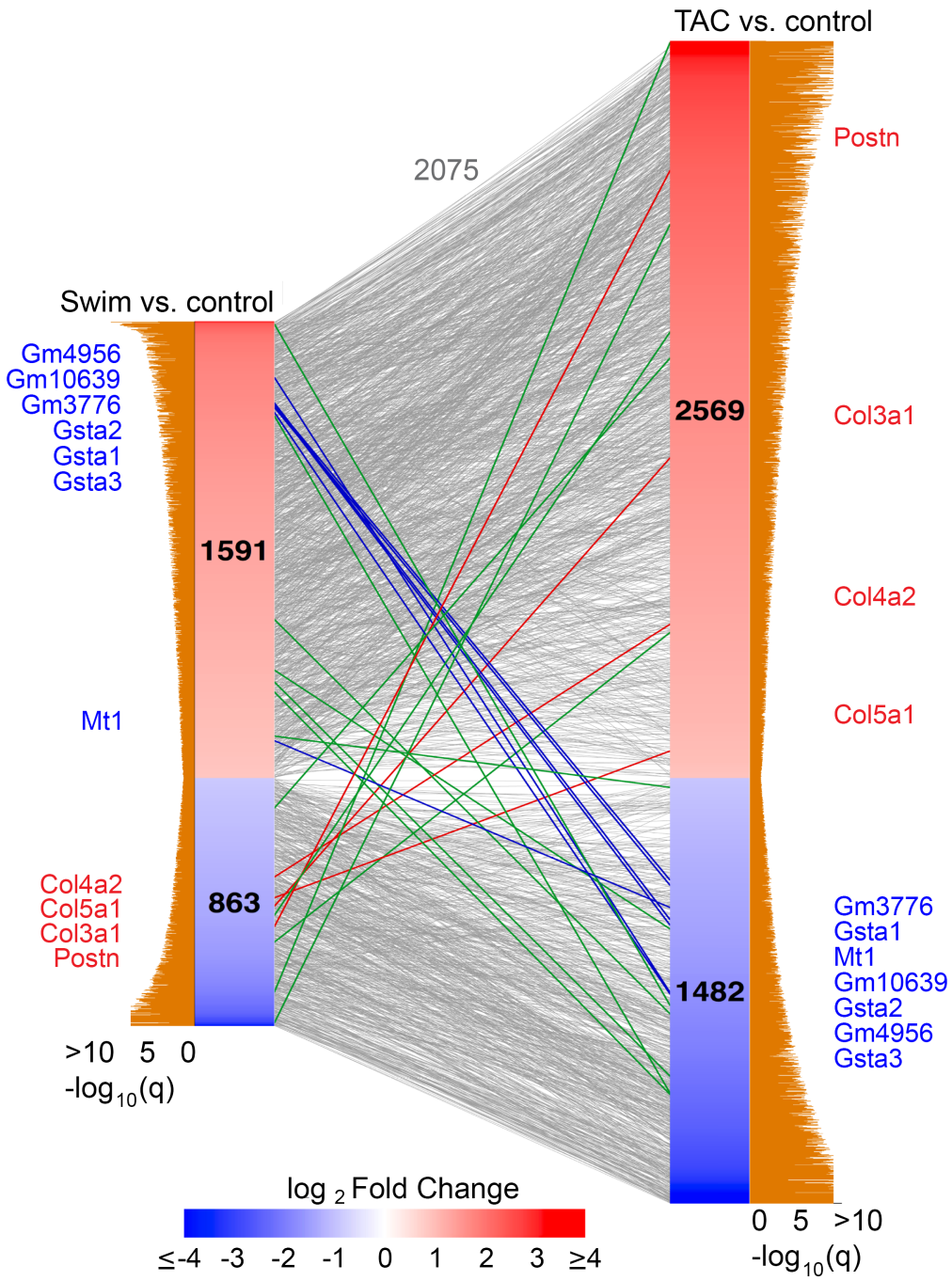

Figure 3. Identifying inversely regulated genes in CFs during pathological versus physiological remodeling. Linear network representation of Venn diagram depicting the relationship of gene expression changes in CFs from C57BL/6 animals between 10 -day swim or 10 -day TAC versus controls. For each list, significantly changed genes $(q<0.05)$ were retained if at least 1 condition attained an FPKM $>1$. The number of genes retained in each condition is indicated in plot and were sorted by their $\log _{2}$ fold change. Corresponding tan bars indicate $-\log _{10} q$ value, and lines between the 2 columns indicate common genes. Lines in blue, red, or green denote the 23 genes displaying expression changes in opposite directions. Detoxification genes that are enriched in swim and downregulated in TAC are represented and identified in blue, and known profibrotic genes that are enriched in TAC and downregulated in swim are represented and identified in red. Green lines indicate inversely expressed genes. Gray lines represent genes in either swim vs. control or TAC vs. control that move in the same direction.

We next asked whether exogenous MT was sufficient to protect isolated neonatal mouse CMs from oxidative stress-induced cell death. In contrast to untreated cells, we observed a dose-dependent reduction in $\mathrm{CM}$ viability upon treatment with $\mathrm{H}_{2} \mathrm{O}_{2}$ (Figure 7G). Addition of either recombinant MT1 or MT2A improved neonatal myocyte viability upon $\mathrm{H}_{2} \mathrm{O}_{2}$ treatment (Figure $7 \mathrm{H}$ ). We detected approximately $25 \%$ $45 \%$ lethality in CMs treated with $125 \mu \mathrm{M}$ or $250 \mu \mathrm{M} \mathrm{H}_{2} \mathrm{O}_{2}$, compared with vehicle-treated CMs (Figure 7, $\mathrm{H}$ and I). Culture with recombinant MT2A led to a dose-dependent rescue of viability in CMs. Survival of CMs treated with $125 \mu \mathrm{M}$ was fully restored with $5 \mu \mathrm{M}$ MT2A, whereas viability of CMs treated with $250 \mu \mathrm{M} \mathrm{H}_{2} \mathrm{O}_{2}$ was only partially restored with $5 \mu \mathrm{M} \mathrm{MT2A}$ (Figure 7I). Furthermore, viability of cultured adult CMs increased when treated with recombinant MT1 or MT2A (Figure 7J). Taken together, this study revealed that MTs, which are produced primarily by nonmyocytes in the heart, can be taken up by CMs in culture, providing a paracrine cardioprotective mechanism at baseline or in response to the excessive metabolic demands of exercise that is suppressed by TGF- $\beta 1 / p 38$ MAPK signaling in disease. 


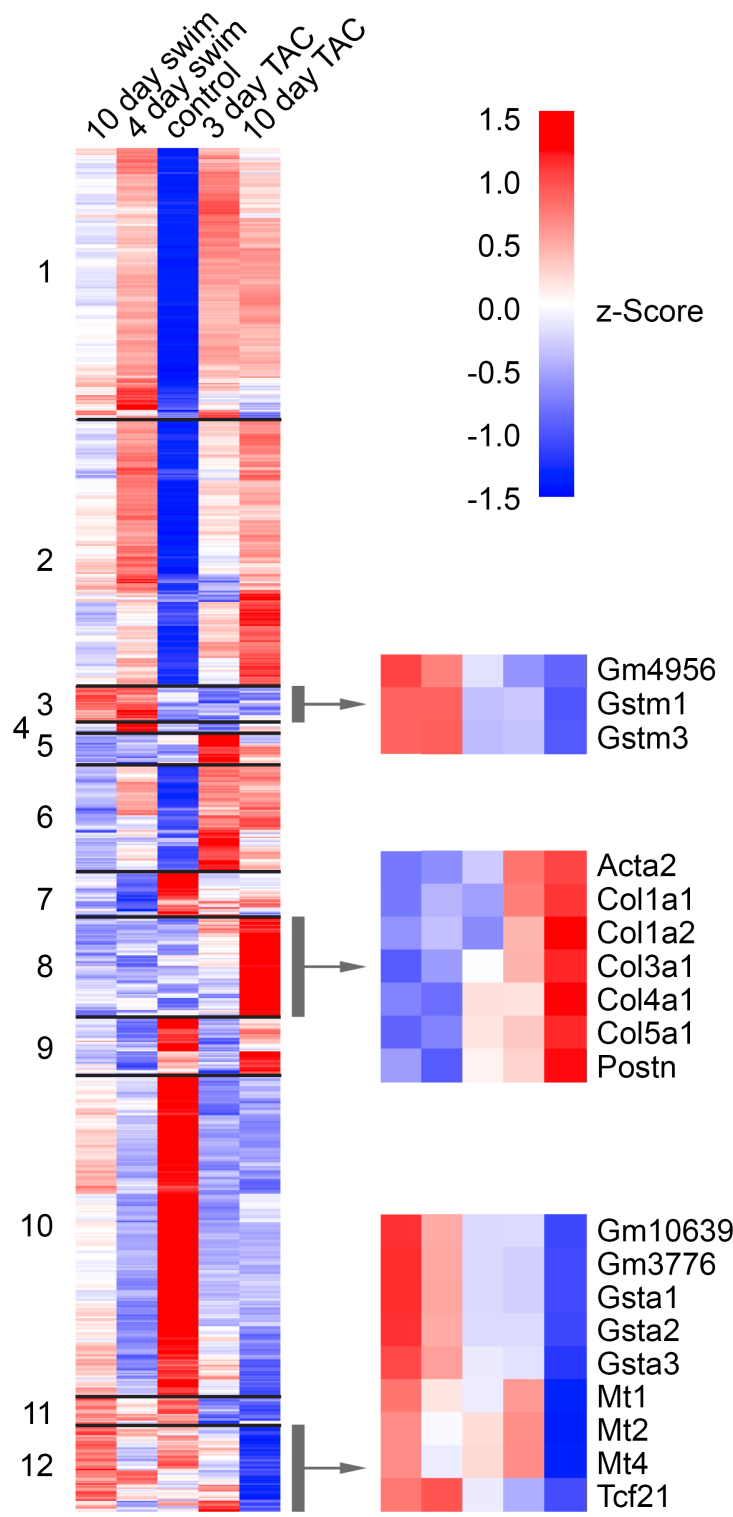

Figure 4. Heat map of genes expressed in swim and TAC. Hierarchical clustering of 4528 genes based upon average FPKM from 10-day swim, 4-day swim, control, 3-day TAC, and 10-day TAC. Select genes are specified for 3 of the 12 clusters with genes that are inversely expressed in swim and TAC. Upregulated genes are represented in red, and downregulated genes are represented in blue.

Metallothionein deletion leads to reduced exercise tolerance. We next asked whether MT1/2 is required to preserve cardiac function in response to the physiological demands of exercise. To answer this question, we subjected mice that lack $M t 1$ and $M t 2$ (MTKO) $(41,42)$ and strain-matched WT controls to a 28-day swim training regimen. MTKO mice had normal cardiac function at baseline and displayed hypertrophic cardiac growth during exercise, as assessed by heart weight/tibia length (HW/TL) ratio (Figure 8A, Supplemental Figure 5A). Decreased expression of $C e b p \beta$ also confirmed physiological remodeling in both WT and MTKO mice during exercise (Supplemental Figure 5, B-E). However, in stark contrast to control mice, MTKO mice developed cardiac dysfunction within 7 days of initiating the swimming regimen, revealed by reduced ejection fraction (EF) and fractional shortening (FS) (Figure 8, B and C). While there was no significant change in CM cross-sectional area, echocardiography revealed that systolic volume was significantly elevated in MTKO animals after 28 days of swim, corresponding to an increased left ventricle mass (LVM) (Figure 8D and Supplemental Figure 5, F and G). Notably, female MTKO mice were more tolerant of exercise than male mice, exhibiting a delay in the onset of cardiac dysfunction (Supplemental Figure 5, H and I). 

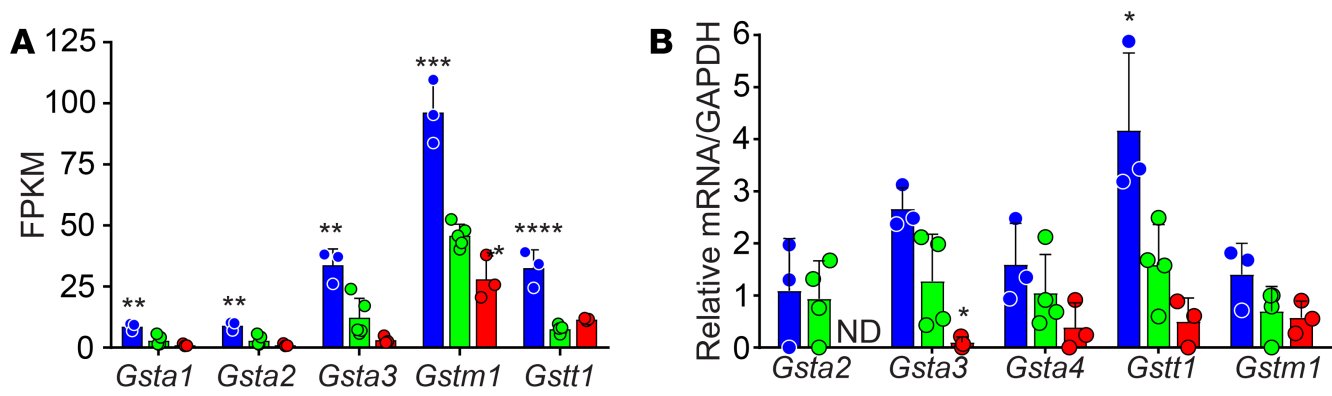

\begin{tabular}{|lll|}
\hline 10 day swim CF & a control CF & day TAC CF \\
& a control CM & day swim CM day TAC CM \\
\hline
\end{tabular}
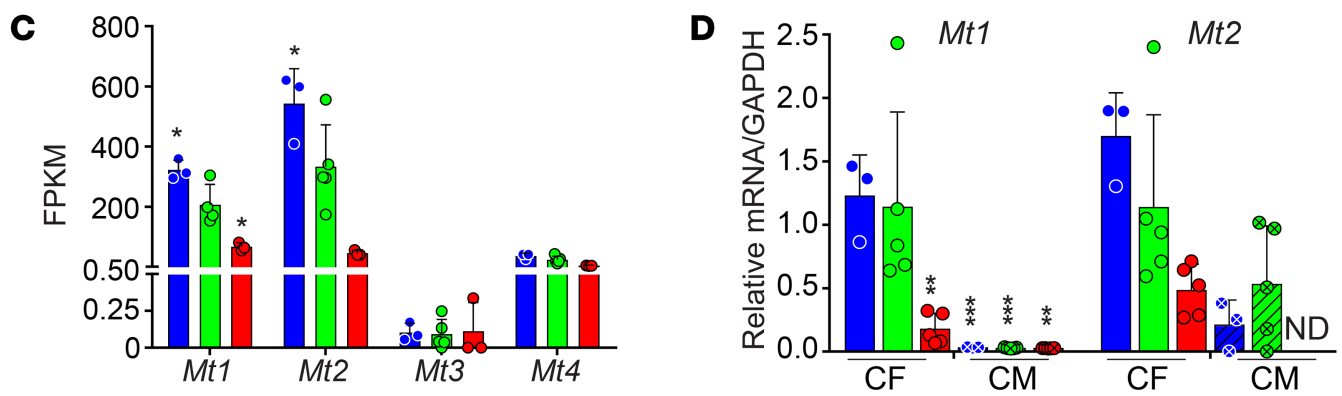

Figure 5. Expression and validation of detoxification pathway genes. FPKM values ( $\mathbf{A}$ and $\mathbf{C})$ and $\mathrm{q} P C R$ validation of RNA-Seq data from C57BL/6 animals (B and $\mathbf{D}$ ) for genes encoding GST family proteins (A and $\mathbf{B}$ ) or MT family proteins (C and $\mathbf{D}$ ) in mouse CFs and CMs after 10-day swim and 10-day TAC, compared with controls. Statistics were performed using 1-way ANOVA and Tukey's post-hoc test $(n=3$ except for control where $n=5)$. ${ }^{*} P<0.05$, ${ }^{* *} P<0.01,{ }^{* *} P<0.001,{ }^{* * *} P<0.0001$.

Although fibrosis was not observed in sedentary MTKO animals and there was no apparent change in PDG$\mathrm{FR}^{+}{ }^{+}$cell number in MTKO animals after 28 days of swim training (Supplemental Figure 5, J-M), quantification of Picrosirius red staining of collagen fibers revealed significant increases in epicardial, perivascular, and interstitial cardiac fibrosis compared with WT following 28 days of swim training (Figure 8, E-H). Furthermore, while vessel density was normal in MTKO mice at baseline, we observed vascular rarefaction in MTKO animals after 28 days of swim training, as revealed by labeling of endothelial cells with isolectin and von Willebrand Factor (vWF) (Figure 8, I and J). Consistent with these indicators of maladaptive remodeling, male MTKO animals develop diastolic dysfunction as indicated by reduced E/A ratio (peak velocity of blood flow in early diastole [ $\mathrm{E}$ wave] to late diastole [A wave]) after 28 days of swimming (Figure $8 \mathrm{~K})$. We also subjected WT and MTKO animals to treadmill running as an alternative form of exercise. MTKO animals fatigued much more quickly than control animals; however, extremely poor voluntary exercise capacity of the $129 / \mathrm{SvJ}$ strain, especially upon MT1/2 deletion, precluded evaluation of physiological cardiac remodeling (Supplemental Figure 5, N-P) (43). Taken together, these data strongly suggest that MTs are required to preserve cardiac function during physiological hypertrophic growth.

Loss of MTs is conserved in human HF fibroblasts. We have described a potentially novel cardioprotective mechanism of CF-derived MTs that is active at rest and during exercise but is lost in disease in a p38-dependent manner. To determine whether the loss of MTs in pathological remodeling is conserved in the failing human heart, we obtained left ventricle tissue from 15 human HF patients and 17 individuals who died of reasons unrelated to heart disease (Supplemental Table 3). MT1 was significantly suppressed, and $M T 2$ trended downward in human HF samples in an inverse relationship to markers of HF such as NPPA and COL1A2 (Figure 9, A-D). This led us to investigate whether the mechanism of p38-dependent repression of $M T 1 / 2$ was conserved in human HF. To this end, we isolated CFs from left ventricle tissue isolated from HF patients undergoing ventricular assist device implantation (age, sex, and health status is included in Supplemental Table 3). Treatment of pathological human CFs with either a pan-p38 inhibitor (BIRB796) or a p38 $\alpha / \beta$-specific inhibitor (SB203580) resulted in a significant increase in $M T 1$ and $M T 2$ expression (Figure 9, $\mathrm{E}$ and $\mathrm{F}$ ). These data suggest that loss of $M T$ expression 
A
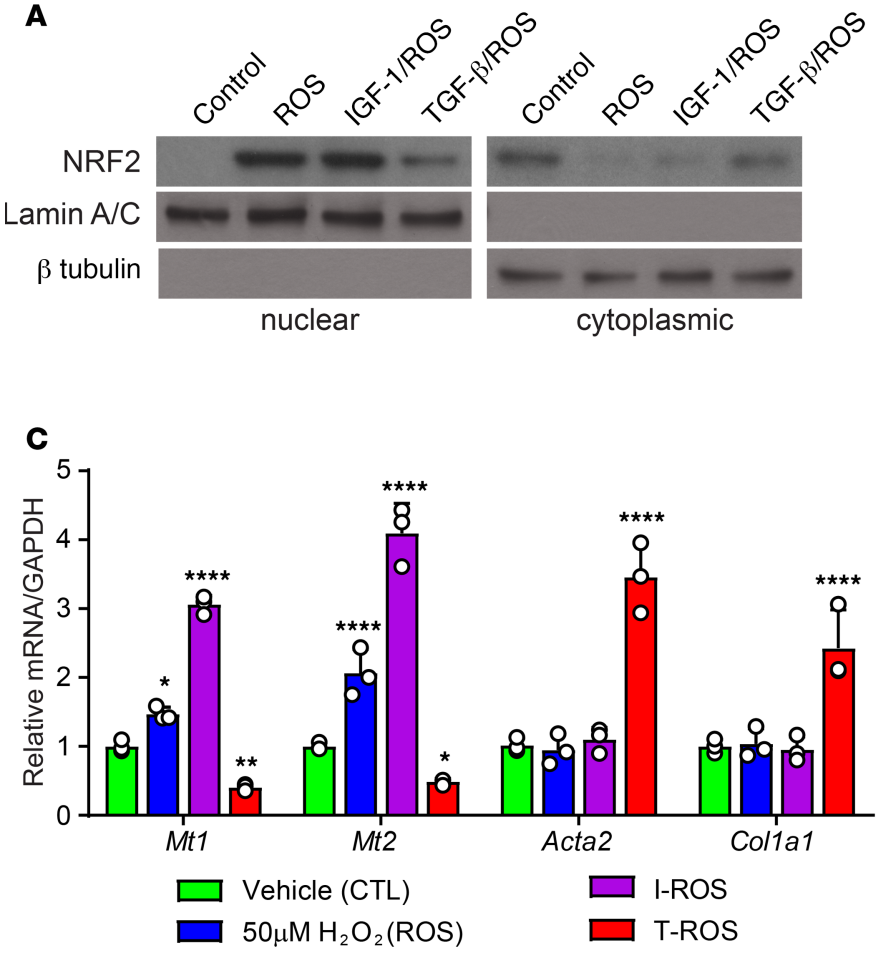
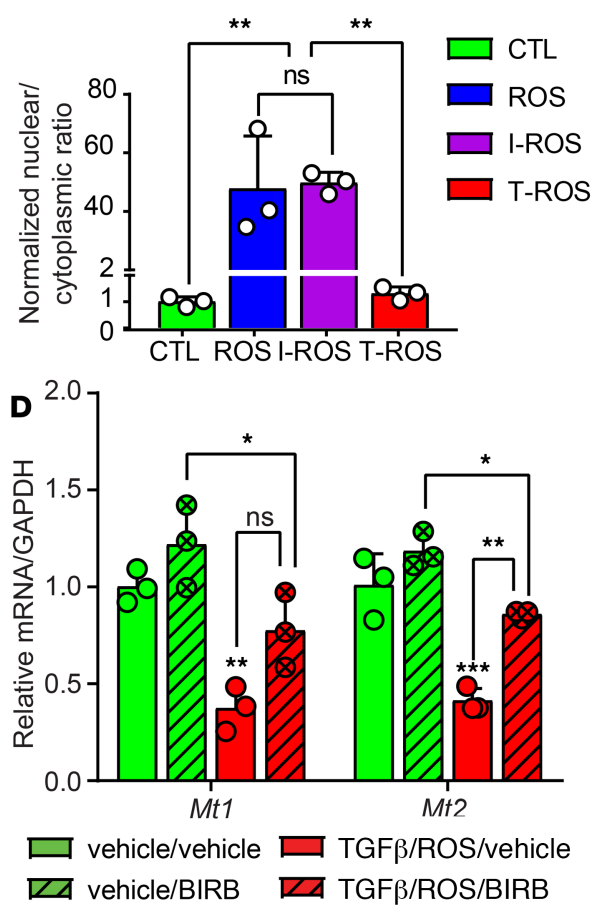

Figure 6. NRF2-dependent toxin and ROS scavenger pathways are suppressed in pathological mouse CFs in a p38-dependent manner. (A) Western blot for NRF2 on nuclear and cytoplasmic protein lysates from neonatal mouse CFs treated with vehicle, ROS (50 $\mu \mathrm{M} \mathrm{H} \mathrm{O}_{2}$ ), ICF-1 (100 ng/mI)/ROS, or TGF- $\beta 1$ (10 ng/ml)/ROS. Lamin A/C and $\beta$-tubulin are used as controls for nuclear and cytoplasmic enrichment, respectively. (B) Quantification represents 3 independent experiments. (C) qPCR analysis of Mt1/2, Acta2, and Col1a1 expression in neonatal CFs treated with ROS, ICF-1/R0S, or

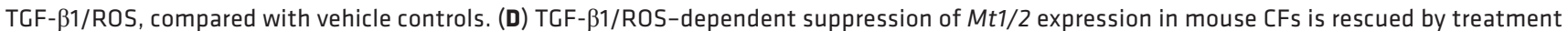
with a pan-p38 inhibitor (BIRB796, $5 \mu \mathrm{M})(n=3$ per treatment). Statistics in B-D were performed using 1-way ANOVA and Tukey's post-hoc test ( $n=3$ ). ${ }^{*} P<0.05,{ }^{* *} P<0.01,{ }^{* *} P<0.001,{ }^{* * * *} P<0.0001$.

in CFs is conserved in human HF and occurs at least partially through noncanonical TGF- $\beta 1-$ mediated p38 MAPK signaling. Our study presents an intriguing paradigm where p38 MAPK signaling inhibits MT-based cardioprotective mechanisms in CFs during disease.

\section{Discussion}

CFs are often studied in the context of heart disease and pathological remodeling; thus, their contribution to cardiac homeostasis in healthy individuals has been largely overlooked. This study reveals for the first time to our knowledge that exercise and disease induce distinct gene expression changes in CFs. NRF2-dependent antioxidant genes, including Mt1/2 and Gst genes, are induced in response to exercise and suppressed in disease. While treatment with recombinant MT1/2 did not dramatically alter the expression of ECM genes in CFs in vitro, mice lacking MTs fail to tolerate strenuous exercise and develop cardiac fibrosis. We found that MTs secreted from fibroblasts are taken up by CMs in vitro and reduce the damaging effects of ROS, emphasizing the complexity of the oxidative stress response. Importantly, suppression of $M T 1 / 2$ expression in pathological cardiac remodeling is conserved in human heart disease, and Mt1/2 expression is restored by TGF- $\beta 1 / \mathrm{p} 38$ MAPK inhibition. Taken together, our study uncovers a potentially novel cardioprotective gene program in CFs that promotes cardiac health and expands upon the nontraditional functions of CFs reported in various aspects of cardiac physiology $(21,22)$ (Supplemental Figure 6).

The primary goal of this study was to utilize transcriptional profiling to characterize the CF phenotype in health and disease. Previous studies have used predefined markers to isolate CFs by flow cytometry in conjunction with genetic lineage tracing methods or immunofluorescence with markers including Thy1, Postn, Tcf21, Colla2, Pdgfro, and Vim (30, 44-48). However, identifying a specific and universal fibroblast marker remains a challenge (49). To identify transcriptional changes in CFs without bias toward 1 specific subpopulation, we utilized a standard differential plating method to isolate 
A

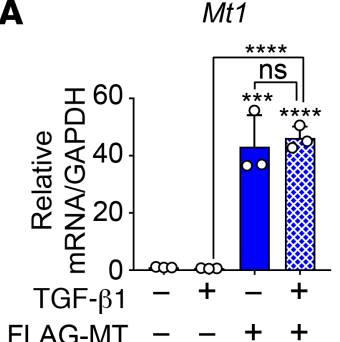

B

Mt2

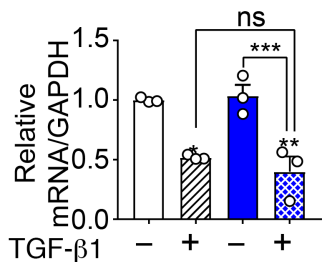

FLAG-MT - - + +

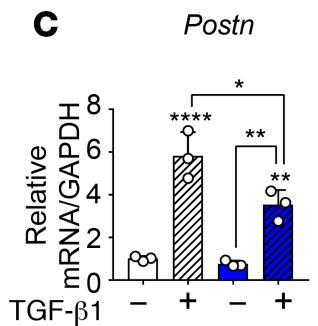

D

Col1a1

E Acta2

FLAG-MT - - + +

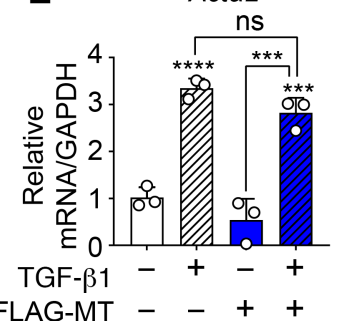

FLAG-MT - - + +
$\mathbf{F}$
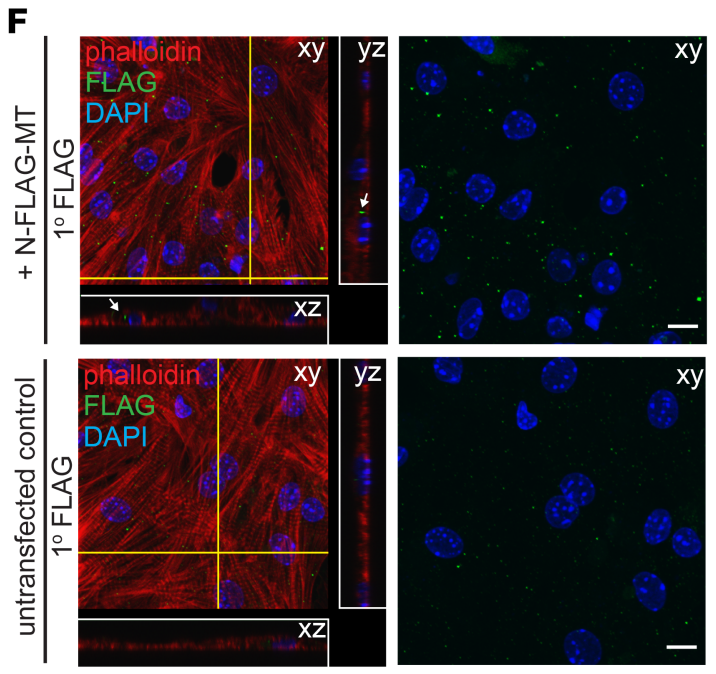
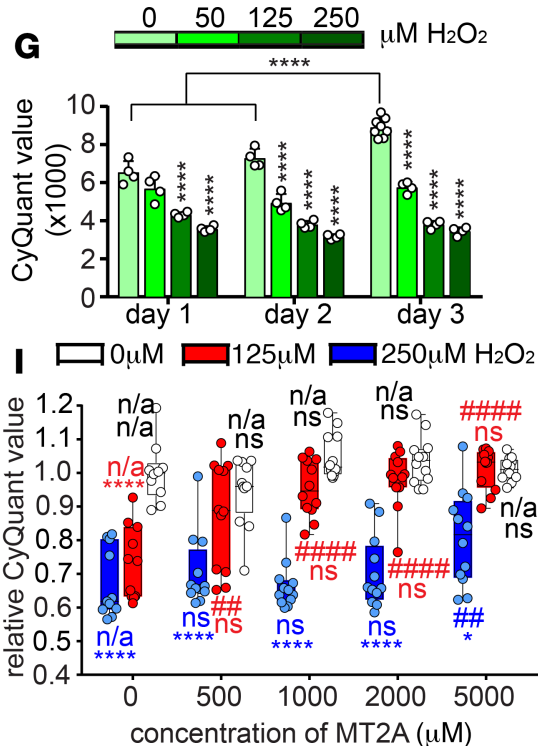
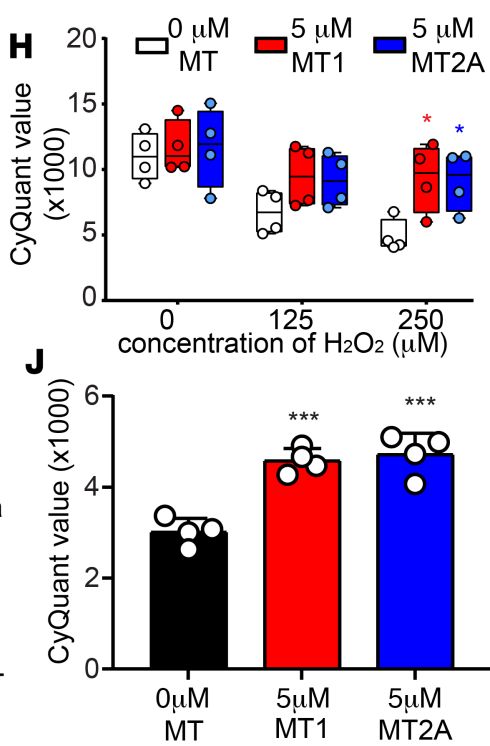

Figure 7. MT modestly inhibits the myofibroblast response and protects CMs from ROS-induced cell death. NIH-3T3 cells were transfected with a plasmid encoding either N-FLAG-MT1 or FLAG and treated with TGF- $\beta 1$ (10 ng/ml) or vehicle. (A-E) qPCR analysis demonstrates minor but significant reduction of Postn expression in MT1-overexpressing cells but not other myofibroblast markers. Statistics were performed using 1-way ANOVA and Tukey's post-hoc test $(n=3) .{ }^{*} P<0.05,{ }^{*} P<0.01,{ }^{*}{ }^{*} P<0.001$, ${ }^{* * *} P<0.0001$. (F) Representative images of neonatal mouse CMs were treated with conditioned media from untransfected NIH-3T3 cells or cells expressing N-FLAG-MT1. Confocal immunofluorescent detection of FLAG localization reveals uptake of N-FLAG-MT1 by CMs (white arrows). Yellow lines on the xy plane indicate orthogonal planes outlined in white (yz and xz). Scale bars: $10 \mu \mathrm{m}$. Experiment was performed 4 independent times. (G) Neonatal mouse CMs were treated with indicated dose of $\mathrm{H}_{2} \mathrm{O}_{2}$, and cell number was assessed by CyQuant DNA-dye incorporation. $\mathrm{H}_{2} \mathrm{O}_{2}$ treated CM displayed reduced viability compared with untreated CMs at the indicated treatment time ( $n=4$ per data point). (H) Neonatal mouse CMs were treated with indicated concentration of $\mathrm{H}_{2} \mathrm{O}_{2}$ and recombinant MT1 or MT2A, followed by CyQuant DNA-dye incorporation to assess cell number. MT1 and MT2A were equally able to protect neonatal CMs from $\mathrm{H}_{2} \mathrm{O}_{2}$-induced cell death ( $n=4$ per data point). (I) Neonatal mouse CMs were cultured in the presence of 0,125 , or $250 \mu \mathrm{M} \mathrm{H}_{2} \mathrm{O}_{2}$ and $0,500,1000,2000$, or $5000 \mathrm{nM}$ of recombinant rabbit MT2A. CyQuant DNA dye incorporation reveals a dose-dependent protection of CMs from cell death by MT2A ( $n=12$ per data point). (J) Treatment of adult CM with $5 \mu M$ MT1 or MT2A improved viability in vitro. Statistics in G-H were performed using 2-way ANOVA and Tukey's post-hoc test ( $n=4$ per data point). ${ }^{*} P<0.05,{ }^{* * *} P<0.001,{ }^{* * * *} P<0.0001$ compared with $0 \mu M$ $\mathrm{H}_{2} \mathrm{O}_{2}$ within indicated MT2A treatment. ${ }^{\# \#} P<0.01$, \#\#\# $P<0.0001$ compared with $\mathrm{O}$ nM MT2A within indicated $\mathrm{H}_{2} \mathrm{O}_{2}$ treatment.

CFs based on their rapid adherence to tissue culture plastic compared with other cardiovascular cell types. Although we also isolated a small percentage of vascular and hematopoietic cells, the adherent nonmyocyte fraction consisted of approximately $80 \%$ fibroblasts, and the percentage of contaminating cells did not change significantly after exercise or disease. Furthermore, we minimized differential plating duration to prevent transcriptional changes that might occur from the mechanical disturbance of extended culture or flow cytometry. Indeed, CFs freshly isolated from mice subjected to various pathophysiological stressors exhibited considerable transcriptional divergence. We interpret this to indicate that our isolation method not only preferentially captures fibroblasts while limiting bias, but minimizes transcriptional changes arising from mechanical disruption.

Resident CFs proliferate in response to pathological stimuli and give rise to activated myofibroblasts (46, 50-52). More recently, a lineage tracing study revealed that Postn and Tcf21 may mark activated and quiescent CFs, respectively (30). Our data demonstrate that exercise promotes the quiescent CF phenotype marked by reduced levels of Postn and key ECM genes, even compared with sedentary control CFs. It is interesting to consider the possibility that physiological signals might be harnessed to revert pathological myofibroblasts to a more quiescent state. 
A

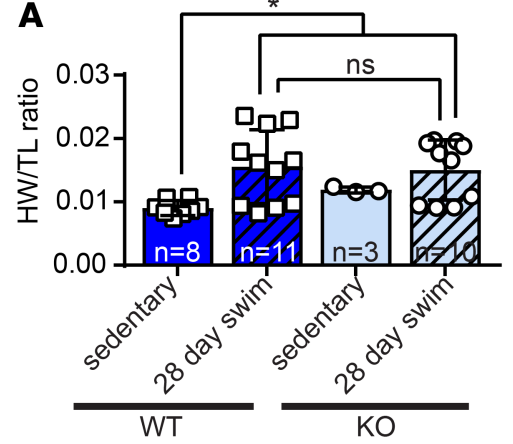

D Systolic Volume

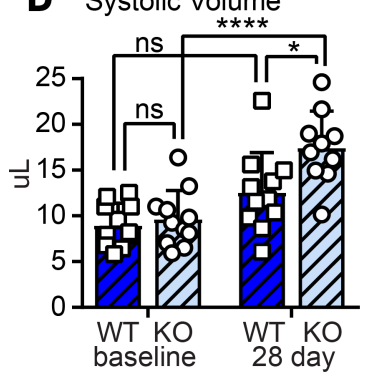

F Interstitial Fibrosis

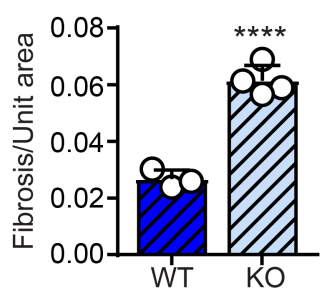

B

E

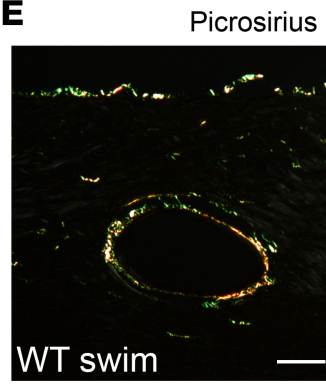

G Epicardial Fibrosis

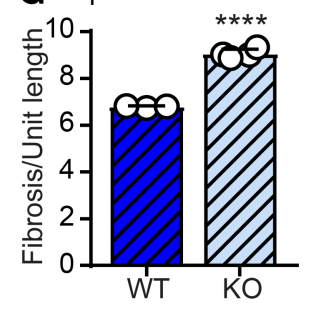

Ejection Fraction

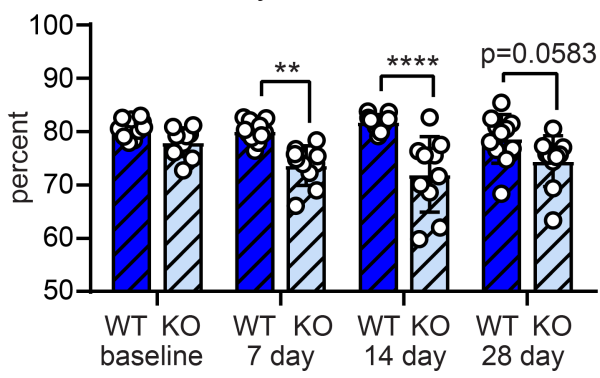

C

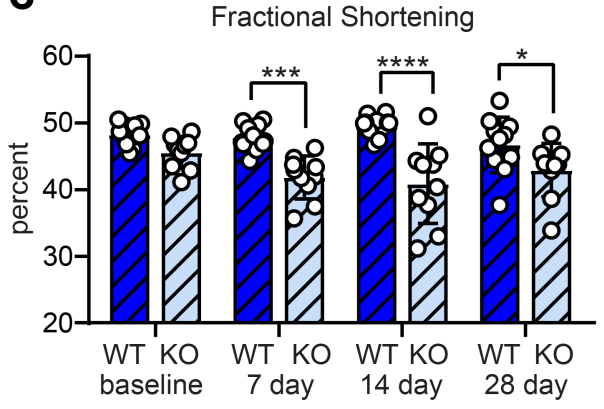

I Isolectin and vWF staining
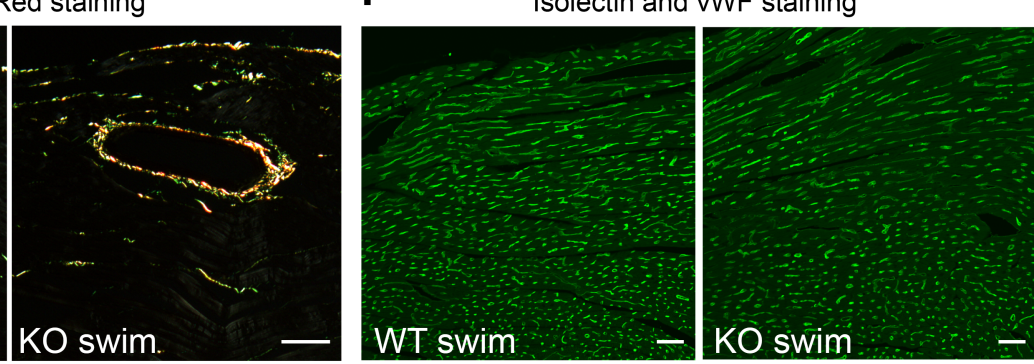

H Perivascular Fibrosis
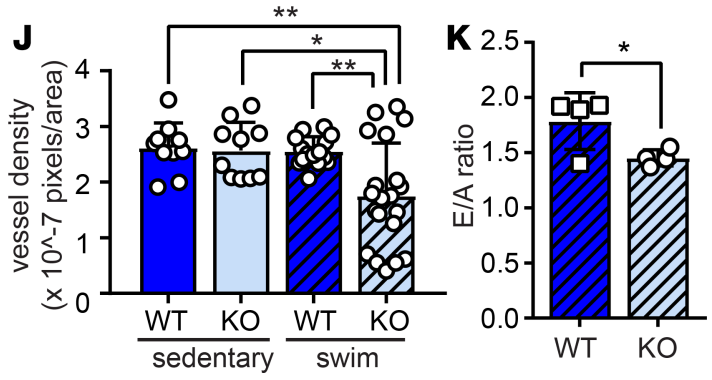

Figure 8. MT1/2 deficiency leads to exercise intolerance and cardiac dysfunction. MT1/2KO (KO) mice or $1295 \mathrm{JJ}$ WT controls were subjected to a 28 day swimming regimen. (A) Heart weight/tibia length (HW/TL) ratio of KO and WT animals reveals cardiac hypertrophy after $28-$ day swim but no significant difference due to loss of MT1/2. Echocardiographic determination of (B) ejection fraction, (C) fractional shortening, and (D) systolic volume indicates that systolic cardiac function is impaired in swim-trained KO mice. (E) Representative polarized light images of Picrosirius red staining of left ventricular free wall (LVFW) to evaluate fibrosis in swim-trained WT and KO animals. WT sedentary, $n=8 ;$ WT swim, $n=11 ; \mathrm{KO}$ sedentary, $n=3$; KO swim, $n=10$. (F-H) Quantification of Picrosirius red staining from similar regions of the LVFW demonstrates increased fibrosis in the (F) interstitial, (G) epicardial, and (H) perivascular regions of $\mathrm{KO}$ animals. Pixel coverage was quantified to indicate area of fibrosis. WT swim, $n=3$; KO swim, $n=5$. (I) Isolectin and von Willebrand Factor costaining of LVFW to evaluate vascularization in swim-trained WT and KO animals. Three to 5 regions of interest were quantified per animal. WT swim, $n=3$; KO swim, $n=5$. (J) Pixel density quantification reveals reduced vascularization in KO animals compared with controls. (K) Echocardiographic analysis of E/A ratio demonstrates impaired diastolic function in swim-trained WT and KO mice, $n=4$. Statistics in F-H and $\mathbf{K}$ were performed using two-tailed Student's $t$ test. Statistics in B-D were performed using 2-way ANOVA and Tukey's post-hoc test. Statistics in A were performed using 1-way ANOVA and Tukey's post-hoc test. ${ }^{*} P<0.05,{ }^{* *} P<0.01,{ }^{* * *} P<0.001,{ }^{* * * *} P<0.0001$. Scale bars: $50 \mu \mathrm{m}$.

Our transcriptomic analysis also revealed that exercise might promote cardiac health by inducing or stabilizing Nfe2l2 expression and the NRF2-dependent antioxidant gene program in CFs. The NRF2-dependent pathway has been previously implicated as a stress-sensitive cytoprotective mechanism that stimulates the expression of genes encoding toxin and ROS scavengers such as Mts (53-55). NRF2 accumulates in the nucleus of CFs treated with ROS or IGF-1/ROS, consistent with induction of the antioxidant gene response by exercise. Importantly, TGF- $\beta 1$ reverses the nuclear accumulation of NRF2 induced by ROS and blunts $M t 1 / 2$ expression. We show that TGF- $\beta 1 /$ p38 MAPK signaling suppresses $M t 1 / 2$ expression in pathological mouse and human CFs. This is consistent with recent reports that p38 signaling is critical for myofibroblast activation and that TGF- $\beta$ inhibition can elevate $M t 1$ expression in skeletal myoblasts $(39,56,57)$. Together, these data might imply a more general link between TGF- $\beta$ / p38 signaling and regulation of NRF2 activity $(56,57)$.

Importantly, we observe decreased expression of MTs in human HF compared with healthy heart tissue, suggesting this cardioprotective mechanism is also lost in human disease. Although previous studies report elevated MT in HF tissue versus the unloaded heart $(58,59)$, differences in study design 
A

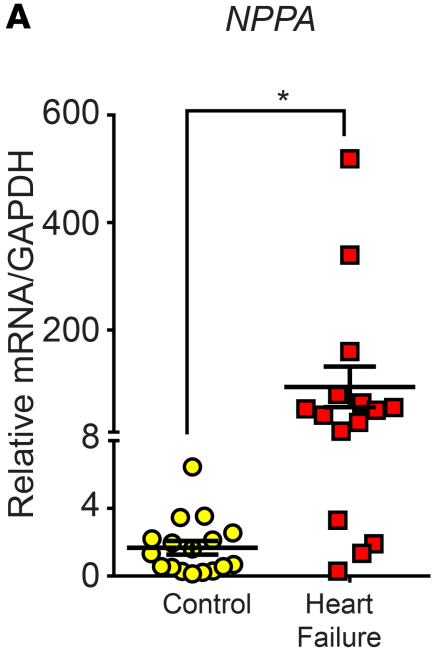

E
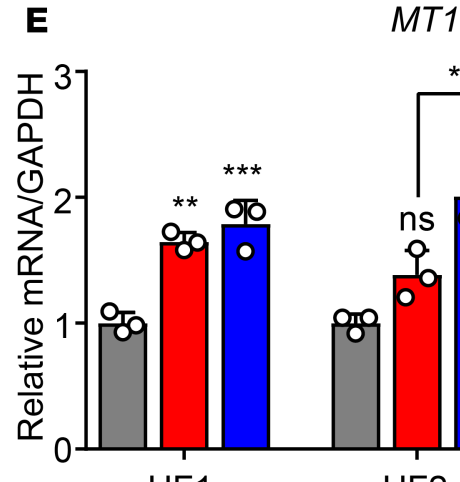

HF1
B

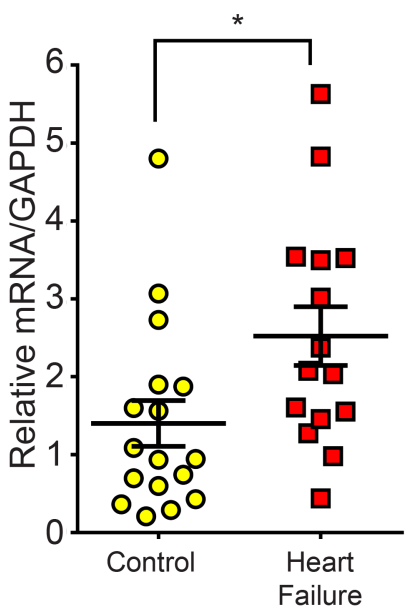

C

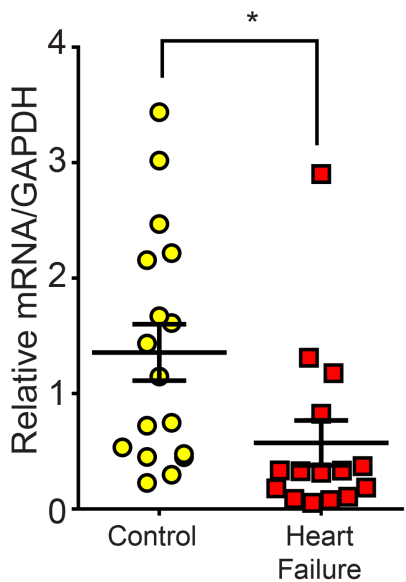

F

(1)

HF3

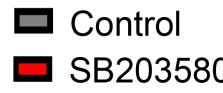
口 BIRB796

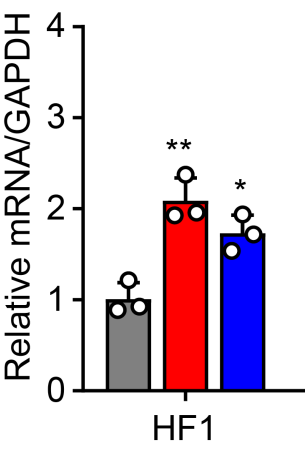

D

MT2

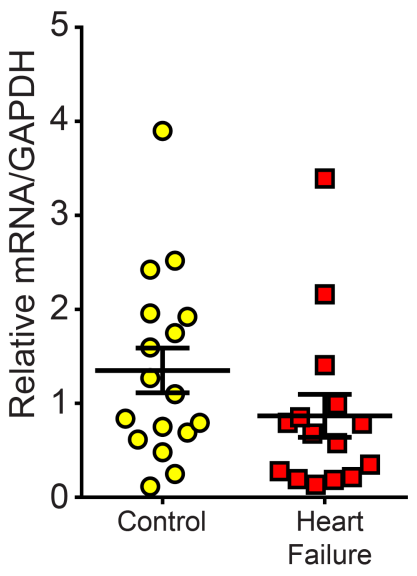

MT2A

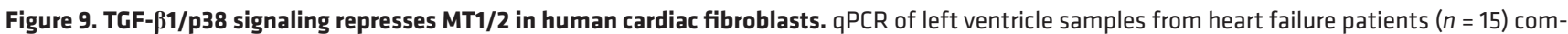
pared with control cardiac tissue $(n=17)$ reveals increased (A) Nppa and (B) Col1a2 expression and reduced (C) Mt1 and (D) Mt2 expression in human HF. Primary human fibroblasts were isolated from cardiac tissue obtained from HF patients undergoing ventricle assist device implantation. qPCR analysis revealed the expression of (E) Mt1 and (F) Mt2a from CFs isolated from individual human heart failure patients and treated with $10 \mu \mathrm{M}$ SB203580 or $5 \mu \mathrm{M}$ BIRB796. Statistics in A-D were performed using two-tailed Student's $t$ test. Data in $\mathbf{E}$ and $\mathbf{F}$ represent the mean \pm SD of $n=3$ technical replicates. ${ }^{*} P<$ $0.05,{ }^{* *} P<0.01,{ }^{* * *} P<0.001$.

preclude a direct comparison; it remains possible that the unloaded heart has less MT than the healthy heart. Importantly, p38 inhibitors induce MT1/2 expression in fibroblasts isolated from human HF patients. This indicates that conservation of p38-dependent myofibroblast activation in mice and humans also extends to regulation of $M T 1 / 2$ expression. It is intriguing to speculate that reducing fibroblast activation by specifically inhibiting p38 may be accompanied by increased $M T$ expression, which may be cardioprotective.

Aerobic exercise in human HF patients has proven to have a number of beneficial outcomes, including improved cardiac function and reduced mortality (60). A number of mouse models have been developed to study the physiological consequences of exercise, including free-wheel running, treadmill running, and swim training, each with advantages and disadvantages. In particular, some mouse strains do not perform well in voluntary running, and treadmill running often requires the use of an electrical stimulus to maintain participation. We chose to study the impact of exercise on the $\mathrm{CF}$ phenotype using a carefully monitored swim training regimen as the exercise model because it is a nonvoluntary exercise regimen that induces reproducible cardiac hypertrophy (5). Previous studies report that exercise ameliorates pathological cardiac remodeling by improving ventricular function and reducing fibrosis $(2,61,62)$. The cardioprotective benefit of exercise partially stems from CEBP-dependent transcriptional changes, which supports $\mathrm{CM}$ viability and regeneration in the face of pathological insult (6). Our study supports these reports and suggests that increased CM viability stimulated by exercise may partially stem from the maintenance of MT1/2 levels in CFs, mirroring the neuroprotection afforded by astrocyte-derived MT (28). In addition, the balance of antioxidant scavengers and ROS is also critical to maintaining CM function (63). We found that MTKO animals exhibit impaired systolic 
and diastolic dysfunction, which may be a function of increased fibrosis and may also be consistent with the ability of increased ROS burden to impair CM contractility and relaxation $(64,65)$. This is consistent with studies in which loss of metallothionein aggravates ischemia/reperfusion-induced fibrosis and hypertrophy, as well as blunts $\mathrm{H}_{2} \mathrm{O}_{2}$-induced fibrosis in vitro $(66,67)$. Together with studies demonstrating that CM-specific overexpression of MTs protects from diabetes-induced oxidative stress and cardiomyopathy, future studies into the role of MTs and ROS in CM calcium handling are warranted (68-70).

Physiological remodeling is normally characterized by coordinated CM growth and increased vascularity $(71,72)$. This contrasts with pathological cardiac hypertrophy in which CM growth outpaces vascularization, leading to localized ischemia $(73,74)$. We find that MTKO mice display vascular rarefaction in response to strenuous exercise, despite normal coronary vessel density at baseline (75). It is possible that CF-derived MTs may promote endothelial cell survival and function, which in turn promotes cardiac health. Of note, p38 inhibition improves endothelial cell survival and promotes angiogenesis, suggesting a potential role for p38 and MTs in vascular cell viability (76). However, p38 inhibition may also promote cardiac hypertrophy, indicating that - although an attractive target - modulation of p38 to treat pathological cardiac remodeling should be approached with caution $(76,77)$.

Our study also highlights intriguing differences in cardiac adaptations between males and females. In contrast to MTKO males that rapidly develop signs of cardiac dysfunction after swim training, MTKO females do not display significant cardiac dysfunction until after 28 days of exercise. We interpret these results to be consistent with previous findings that female mice exhibit a more robust hypertrophic response to exercise than males, which may underlie extended exercise tolerance observed even in the absence of MTs $(43,78)$.

Although considerable effort has been devoted to harnessing the cardioprotective benefits of antioxidants, therapeutic efficacy has not proven successful. Likely reasons include poor bioavailability of systemically administered antioxidants, limited intracellular therapeutic targets, and an incomplete understanding of the complex mechanisms that regulate cardiomyopathic oxidative stress $(79,80)$. Ultimately, prolonged cardiac stress increases oxidative damage from mitochondrial oxygen consumption, resulting in $\mathrm{CM}$ death, $\mathrm{CF}$ activation, and fibrosis. In light of mounting evidence that CFs modulate both ECM dynamics and the response of CMs to their environment, our findings may support a therapeutic strategy based on promoting the physiological CF state to maintain cardioprotective mechanisms in disease.

\section{Methods}

Supplemental Methods are available online with this article.

TAC, MI, and swim models. After administering anesthesia, C57BL/6 animals were subjected to TAC, which was performed using a 27-gauge needle. Sham surgeries were performed by passing the suture around the aorta without ligating. MI was performed by intramural ligation of the left anterior descending coronary artery $2 \mathrm{~mm}$ from its origin. The swim model was performed in 12- to 14-week-old C57BL/6, 129SvJ, or MTKO animals as previously described (3) with slight modifications.

Primary cell isolation. Adult mouse ventricular fibroblasts were isolated by Langendorff perfusion to obtain a single-cell suspension. CFs were not passaged before use and were instead isolated by differential plating for 2 hours at $37^{\circ} \mathrm{C}$ and washing the attached cells (CFs) with $1 \times$ PBS before RNA isolation.

Neonatal mouse ventricular fibroblasts and CMs were isolated from P0 neonatal hearts digested at $37^{\circ} \mathrm{C}$ in $0.8 \mathrm{mg} / \mathrm{ml}$ collagenase II (Worthington Biochemicals). Neonatal ventricular fibroblasts were isolated by differential plating. Neonatal CMs were washed from the adherent CFs and plated on gelatin-coated tissue culture plastic.

Human CFs were isolated from heart tissue normally discarded from left ventricle assist device implantation at the University of Rochester Medical Center, with IRB approval and patient consent. Heart tissue was dissociated using a Miltenyi mouse neonatal heart dissociation kit, and viable human CFs were isolated by differential plating for 2 hours at $37^{\circ} \mathrm{C}$. Cells were passaged an average of $3-5$ times before use but always used before P7.

Flow cytometry. Adherent adult CFs were isolated from 3 separate hearts and plated on untreated tissue culture plastic for 3 hours at $37^{\circ} \mathrm{C}$. Nonadherent cells were then removed by washing, and adherent cells were trypsinzed and incubated with directly conjugated antibodies against CD31-PE-Cy7 (BD Pharmingen, 561410), CD45-v450 (BD Pharmingen, 560501), and unconjugated goat anti-mouse PDGFR $\beta$ (R\&D Systems, AF1042) on ice for 1 hour in $\%$ BSA/PBS. Cells were washed with $500 \mu 12 \%$ BSA/PBS, pelleted, and incubated with Alexa 647 donkey anti-goat secondary antibody (Jackson ImmunoResearch, 705-605-147) 
for 30 minutes at room temperature. Cells were then washed with $500 \mu 12 \% B S A / P B S$, resuspended in 200 $\mu 12 \% B S A / P B S$, and sorted through a FACSAria (BD Biosciences). RNA was isolated from the sorted cell populations using Trizol and analyzed by $\mathrm{qPCR}$. Cells were not passaged prior to flow analysis.

RNA analysis. RNA was isolated from CFs and CMs by Trizol/chloroform isolation and genomic DNA removed with Turbo DNAse (Ambion). cDNA was generated from 500-1000 ng RNA using iScript Reverse Transcriptase kit (Bio-Rad) as per manufacturer's recommendations. qPCR was performed with BioRad SYBR-Green master mix on a BioRad CFX Connect per manufacturer's recommendations. Primers used for qPCR are listed in Supplemental Table 2.

Stranded mRNA-Seq. Total CF RNA from control, swim, TAC, or MI C57BL/6 animals was isolated using the RNeasy Plus Kit, and genomic DNA was removed using gDNA columns (Qiagen) per manufacturer's recommendations. RNA concentration and quality was assessed with the Agilent Bioanalyzer (Agilent). Library construction was performed using the TruSeq Stranded mRNA Sample Preparation Kit (Illumina) per manufacturer's recommendation and sequenced using an Illumina HiSeq2500 with a read depth of 20-25 million reads at $1 \times 100 \mathrm{bp}$. Analysis and visualization of derived next generation sequencing (NGS) data was performed with Matlab (The Mathworks) and PRISM (GraphPad). The RNA-Seq data from this publication have been submitted to the Gene Expression Omnibus (GEO) database (accession number GSE89885).

Antibodies. Primary antibodies for immunofluorescence were as follows: mouse anti-FLAG M2 (MilliporeSigma, F1804), mouse IgG (Jackson ImmunoResearch, 015-000-003), FITC-conjugated isolectin B4 (MilliporeSigma, L2879), and FITC-conjugated sheep anti-vWF (Abcam, ab8822). Secondary antibodies used for immunofluorescence were Alexa 488 goat anti-mouse (Invitrogen, A11001), Alexa 594 goat anti-phalloidin (Invitrogen, A12381), and DAPI (Invitrogen, D1306). Primary antibodies used for Western blotting were as follows: ubiquitin (Cell Signaling Technologies [CST], 3936), phospho-p38 (CST, 9211), total p38 (CST, 9212), phospho-AKT (CST, 9271), AKT (CST, 9272), phospho-Smad (CST, 3101), Smad3 (CST, 3103), lamin A/C (CST, 4777), GAPDH (MilliporeSigma, MAB374), NRF2 (Abcam, ab31163), $\beta I I I-t u b u l i n$ (Abcam, ab52901). Full uncut blots are shown in the supplemental material.

MT peptide rescue. Neonatal mouse CMs were isolated and plated at 50,000 cells/well in gelatin-coated 96-well black-sided tissue culture plates. Twenty-four hours later, unattached CMs were washed off, and the surviving CMs were pretreated overnight with designated concentrations of full-length rabbit MT2A (Enzo). Two days after isolation, CMs were treated with 0 , 125, or $250 \mu \mathrm{M} \mathrm{H}_{2} \mathrm{O}_{2}$ (MilliporeSigma) with or without full-length rabbit MT2A for 1 hour at $37^{\circ} \mathrm{C}$. Myocytes were then briefly washed once and cultured in normal neonatal $\mathrm{CM}$ media for 24 hours without $\mathrm{H}_{2} \mathrm{O}_{2}$ or $\mathrm{MT} 2 \mathrm{~A}$ at $37^{\circ} \mathrm{C}$ before measuring cell viability using CyQuant assay (Invitrogen) on a BMG FluoSTAR OPTIMA plate reader per manufacturer's instructions.

Cell culture. NIH-3T3 cells were transfected 18 hours after plating with $15 \mu \mathrm{g}$ of plasmid DNA (pcDNA3.1-N-FLAG-MT or -N-FLAG) using Mirus X2 Transfection Reagent per manufacturer's protocol. For conditioned media experiments, transfected NIH-3T3 cells were cultured in serum-free neonatal CM media with $10 \mu \mathrm{M}$ MG-132 for 24 hours. Conditioned media was then concentrated to $350 \mu 1$ in $3 \mathrm{kDa}$ Ambion concentrators. Neonatal mouse CMs were isolated and plated in laminin-coated MAT-TEK glass bottom petri dishes for 48-72 hours. CMs were then cultured with concentrated conditioned media from transfected NIH-3T3s for 4 hours at $37^{\circ} \mathrm{C}$, fixed, and stained using a standard immunofluorescence protocol. For myofibroblast response experiments, NIH-3T3s were treated with 10 ng/ml TGF- $\beta 124$ hours after transfection with FLAG-MT1, and RNA was collected 24 hours after treatment.

Immunofluorescence and fibrosis staining. Immunofluorescence was performed on CMs after permeabilization with $0.5 \%$ saponin, visualized by confocal microscopy using an Olympus Fluoview FV1000. Vascularization on blinded MTKO or WT heart sections was quantified using ImageJ.

Tissue fibrosis was assessed using either Picrosirius Red (Abcam, ab150681) as per manufacturer's instruction or standard Masson's trichrome stain, blinded, imaged using an Olympus BX51, and quantified using ImageJ (NIH).

Statistics. Statistical analyses were performed using Graphpad Prism 7. Unless otherwise noted, statistics were performed using 1-way ANOVA and Tukey's post-hoc test. Each $P$ value was adjusted for multiple comparisons. Sample sizes were determined by the criterion of reaching adequate power with an effect probability $(\alpha)$ of 0.05 . Data represents the mean \pm SD.

Study approval. All animal experiments were approved by the University Committee on Animal Resources at the University of Rochester. Homozygous MTKO animals on a $129 \mathrm{SvJ}$ background were described 
previously and obtained from the Jackson Laboratory (catalog 002211) (42). 129SvJ and C57BL/6 animals were also obtained from the Jackson Laboratory.

Human heart tissue was obtained from the Kaufman Center for Heart Failure Tissue Bank at Cleveland Clinic or the University of Rochester Medical Center (URMC) with IRB approval and patient consent prior to inclusion in the study. URMC approval no. 52958, Cleveland Clinic approval no. 2378. A total of 35 patient samples were used in this study, with 17 control patients who died for reasons unrelated to cardiac disease and 18 patients who met the clinical requirements for either ventricle assist device implantation or heart transplant. Of these 35 patients, 13 were female and 22 were male, ranging in age from 30-67 years (Supplemental Table 3).

\section{Author contributions}

JKL and EMS conceived the project, designed experiments, analyzed data, and wrote the manuscript. CSM and JDA contributed human patient samples. AA and AR performed bioinformatic analyses. JKL, $\mathrm{RMB}, \mathrm{LSV}$, and RAD carried out all experiments.

\section{Acknowledgments}

This work was supported by grants from the NIH/NHLBI to EMS (R01 HL133761 and R01 HL120919), JKL (5T32HL066988-15), and RMB (5T32HL007937 and F32HL136066). We also acknowledge support from the American Heart Association to EMS (SDG4350046) and JKL (15POST25550114), as well as a Pilot Study grant from the Aab Cardiovascular Research Institute at the University of Rochester School of Medicine and Dentistry. We are grateful to J.M. Miano, C.J. Lowenstein, and members of the Small lab, particularly M.A. Trembley and P. Quijada, for critical reading of the manuscript and helpful suggestions; we are also grateful to O. Slivano of the Aab CVRI Histology Core, C. Morrell and D. Mickelsen of the CVRI Microsurgery Core, and J. Ashton and J. Myers of the URMC Genomics Research Center for expert technical support.

Address correspondence to: Eric Small, Aab Cardiovascular Research Institute, Department of Medicine, University of Rochester School of Medicine and Dentistry, 601 Elmwood Avenue, Box CVRI, Rochester, NY 14642, USA. Phone: 585.276.7706; Email: eric_small@urmc.rochester.edu.

1. Hill JA, Olson EN. Cardiac plasticity. N Engl J Med. 2008;358(13):1370-1380.

2. McMullen JR, Jennings GL. Differences between pathological and physiological cardiac hypertrophy: novel therapeutic strategies to treat heart failure. Clin Exp Pharmacol Physiol. 2007;34(4):255-262.

3. Kaplan ML, et al. Cardiac adaptations to chronic exercise in mice. Am J Physiol. 1994;267(3 Pt 2):H1167-H1173.

4. Young DR, et al. Effects of physical activity and sedentary time on the risk of heart failure. Circ Heart Fail. 2014;7(1):21-27.

5. McMullen JR, et al. Protective effects of exercise and phosphoinositide 3-kinase(p110alpha) signaling in dilated and hypertrophic cardiomyopathy. Proc Natl Acad Sci USA. 2007;104(2):612-617.

6. Boström P, et al. C/EBP $\beta$ controls exercise-induced cardiac growth and protects against pathological cardiac remodeling. Cell. 2010;143(7):1072-1083.

7. Davis J, Burr AR, Davis GF, Birnbaumer L, Molkentin JD. A TRPC6-dependent pathway for myofibroblast transdifferentiation and wound healing in vivo. Dev Cell. 2012;23(4):705-715.

8. Haudek SB, et al. Rho kinase-1 mediates cardiac fibrosis by regulating fibroblast precursor cell differentiation. Cardiovasc Res. 2009;83(3):511-518.

9. Small EM, et al. Myocardin-related transcription factor-a controls myofibroblast activation and fibrosis in response to myocardial infarction. Circ Res. 2010;107(2):294-304.

10. Williams SM, et al. Class I HDACs regulate angiotensin II-dependent cardiac fibrosis via fibroblasts and circulating fibrocytes. J Mol Cell Cardiol. 2014;67:112-125.

11. Lighthouse JK, Small EM. Transcriptional control of cardiac fibroblast plasticity. J Mol Cell Cardiol. 2016;91:52-60.

12. Hinz B. Formation and function of the myofibroblast during tissue repair. J Invest Dermatol. 2007;127(3):526-537.

13. Wynn TA. Cellular and molecular mechanisms of fibrosis. J Pathol. 2008;214(2):199-210.

14. Small EM. The actin-MRTF-SRF gene regulatory axis and myofibroblast differentiation. J Cardiovasc Transl Res. 2012;5(6):794-804.

15. Takeda N, et al. Cardiac fibroblasts are essential for the adaptive response of the murine heart to pressure overload. J Clin Invest. 2010;120(1):254-265.

16. Oka T, et al. Genetic manipulation of periostin expression reveals a role in cardiac hypertrophy and ventricular remodeling. Circ Res. 2007;101(3):313-321.

17. Kakkar R, Lee RT. Intramyocardial fibroblast myocyte communication. Circ Res. 2010;106(1):47-57.

18. Ieda M, et al. Cardiac fibroblasts regulate myocardial proliferation through beta1 integrin signaling. Dev Cell. 2009;16(2):233-244.

19. Furtado MB, et al. Cardiogenic genes expressed in cardiac fibroblasts contribute to heart development and repair. Circ Res. 2014;114(9):1422-1434. 
20. Bujak M, Frangogiannis NG. The role of TGF-beta signaling in myocardial infarction and cardiac remodeling. Cardiovasc Res. 2007;74(2):184-195.

21. Martin ML, Blaxall BC. Cardiac intercellular communication: are myocytes and fibroblasts fair-weather friends? J Cardiovasc Transl Res. 2012;5(6):768-782.

22. Souders CA, Bowers SL, Baudino TA. Cardiac fibroblast: the renaissance cell. Circ Res. 2009;105(12):1164-1176.

23. Zhou Y, et al. Inhibition of mechanosensitive signaling in myofibroblasts ameliorates experimental pulmonary fibrosis. $J$ Clin Invest. 2013;123(3):1096-1108.

24. Haak AJ, et al. Targeting the myofibroblast genetic switch: inhibitors of myocardin-related transcription factor/serum response factor-regulated gene transcription prevent fibrosis in a murine model of skin injury. J Pharmacol Exp Ther. 2014;349(3):480-486.

25. Velasquez LS, et al. Activation of MRTF-A-dependent gene expression with a small molecule promotes myofibroblast differentiation and wound healing. Proc Natl Acad Sci USA. 2013;110(42):16850-16855

26. Sandbo N, Kregel S, Taurin S, Bhorade S, Dulin NO. Critical role of serum response factor in pulmonary myofibroblast differentiation induced by TGF-beta. Am J Respir Cell Mol Biol. 2009;41(3):332-338.

27. Sato M, Bremner I. Oxygen free radicals and metallothionein. Free Radic Biol Med. 1993;14(3):325-337.

28. Miyazaki I, et al. Astrocyte-derived metallothionein protects dopaminergic neurons from dopamine quinone toxicity. Glia. 2011;59(3):435-451.

29. Lacy SH, et al. Human lung fibroblasts produce proresolving peroxisome proliferator-activated receptor- $\gamma$ ligands in a cyclooxygenase-2-dependent manner. Am J Physiol Lung Cell Mol Physiol. 2016;311(5):L855-L867.

30. Kanisicak O, et al. Genetic lineage tracing defines myofibroblast origin and function in the injured heart. Nat Commun. 2016;7:12260

31. Crider BJ, Risinger GM, Haaksma CJ, Howard EW, Tomasek JJ. Myocardin-related transcription factors A and B are key regulators of TGF- $\beta 1$-induced fibroblast to myofibroblast differentiation. J Invest Dermatol. 2011;131(12):2378-2385

32. Nebert DW, Vasiliou V. Analysis of the glutathione S-transferase (GST) gene family. Hum Genomics. 2004;1(6):460-464.

33. Raza H. Dual localization of glutathione S-transferase in the cytosol and mitochondria: implications in oxidative stress, toxicity and disease. FEBS J. 2011;278(22):4243-4251.

34. Andrews GK. Regulation of metallothionein gene expression by oxidative stress and metal ions. Biochem Pharmacol. 2000;59(1):95-104.

35. Lacher SE, Lee JS, Wang X, Campbell MR, Bell DA, Slattery M. Beyond antioxidant genes in the ancient Nrf2 regulatory net work. Free Radic Biol Med. 2015;88(Pt B):452-465.

36. Ikeda $\mathrm{H}$, et al. Interaction of myocardial insulin receptor and IGF receptor signaling in exercise-induced cardiac hypertrophy. $J$ Mol Cell Cardiol. 2009;47(5):664-675.

37. Dingar D, et al. Effect of pressure overload-induced hypertrophy on the expression and localization of p38 MAP kinase isoforms in the mouse heart. Cell Signal. 2010;22(11):1634-1644.

38. Woeller CF, O'Loughlin CW, Roztocil E, Feldon SE, Phipps RP. Salinomycin and other polyether ionophores are a new class of antiscarring agent. J Biol Chem. 2015;290(6):3563-3575

39. Molkentin JD, et al. Fibroblast-Specific Genetic Manipulation of p38 Mitogen-Activated Protein Kinase In Vivo Reveals Its Central Regulatory Role in Fibrosis. Circulation. 2017;136(6):549-561.

40. Chung RS, et al. Redefining the role of metallothionein within the injured brain: extracellular metallothioneins play an important role in the astrocyte-neuron response to injury. J Biol Chem. 2008;283(22):15349-15358.

41. Fu Z, Guo J, Jing L, Li R, Zhang T, Peng S. Enhanced toxicity and ROS generation by doxorubicin in primary cultures of cardiomyocytes from neonatal metallothionein-I/II null mice. Toxicol In Vitro. 2010;24(6):1584-1591.

42. Masters BA, Kelly EJ, Quaife CJ, Brinster RL, Palmiter RD. Targeted disruption of metallothionein I and II genes increases sensitivity to cadmium. Proc Natl Acad Sci USA. 1994;91(2):584-588.

43. Lightfoot JT, et al. Strain screen and haplotype association mapping of wheel running in inbred mouse strains. $J$ Appl Physiol. 2010;109(3):623-634.

44. Willis RA, Nussler AK, Fries KM, Geller DA, Phipps RP. Induction of nitric oxide synthase in subsets of murine pulmonary fibroblasts: effect on fibroblast interleukin-6 production. Clin Immunol Immunopathol. 1994;71(2):231-239.

45. Pinto AR, et al. Revisiting Cardiac Cellular Composition. Circ Res. 2016;118(3):400-409.

46. Moore-Morris T, et al. Resident fibroblast lineages mediate pressure overload-induced cardiac fibrosis. J Clin Invest. 2014;124(7):2921-2934.

47. Kong P, Christia P, Saxena A, Su Y, Frangogiannis NG. Lack of specificity of fibroblast-specific protein 1 in cardiac remodeling and fibrosis. Am J Physiol Heart Circ Physiol. 2013;305(9):H1363-H1372.

48. Goodpaster T, Legesse-Miller A, Hameed MR, Aisner SC, Randolph-Habecker J, Coller HA. An immunohistochemical method for identifying fibroblasts in formalin-fixed, paraffin-embedded tissue. J Histochem Cytochem. 2008;56(4):347-358.

49. Kalluri R, Zeisberg M. Fibroblasts in cancer. Nat Rev Cancer. 2006;6(5):392-401.

50. Braitsch CM, Kanisicak O, van Berlo JH, Molkentin JD, Yutzey KE. Differential expression of embryonic epicardial progenitor markers and localization of cardiac fibrosis in adult ischemic injury and hypertensive heart disease. J Mol Cell Cardiol. 2013;65:108-119.

51. Zeisberg EM, et al. Endothelial-to-mesenchymal transition contributes to cardiac fibrosis. Nat Med. 2007;13(8):952-961.

52. Acharya A, et al. The bHLH transcription factor Tcf21 is required for lineage-specific EMT of cardiac fibroblast progenitors. Development. 2012;139(12):2139-2149.

53. Durnam DM, Palmiter RD. Transcriptional regulation of the mouse metallothionein-I gene by heavy metals. J Biol Chem. 1981;256(11):5712-5716.

54. LaRochelle $\mathrm{O}$, et al. Nuclear factor-1 and metal transcription factor-1 synergistically activate the mouse metallothionein-1 gene in response to metal ions. J Biol Chem. 2008;283(13):8190-8201.

55. Tao G, et al. Pitx2 promotes heart repair by activating the antioxidant response after cardiac injury. Nature. 2016;534(7605):119-123

56. Accornero F, Kanisicak O, Tjondrokoesoemo A, Attia AC, McNally EM, Molkentin JD. Myofiber-specific inhibition of TGF $\beta$ signaling protects skeletal muscle from injury and dystrophic disease in mice. Hum Mol Genet. 2014;23(25):6903-6915. 
57. Naidu S, Vijayan V, Santoso S, Kietzmann T, Immenschuh S. Inhibition and genetic deficiency of p38 MAPK up-regulates heme oxygenase-1 gene expression via Nrf2. J Immunol. 2009;182(11):7048-7057.

58. Baba HA, et al. Reversal of metallothionein expression is different throughout the human myocardium after prolonged left-ventricular mechanical support. J Heart Lung Transplant. 2000;19(7):668-674.

59. Blaxall BC, Tschannen-Moran BM, Milano CA, Koch WJ. Differential gene expression and genomic patient stratification following left ventricular assist device support. J Am Coll Cardiol. 2003;41(7):1096-1106

60. Wei X, Liu X, Rosenzweig A. What do we know about the cardiac benefits of exercise? Trends Cardiovasc Med. 2015;25(6):529-536

61. Konhilas JP, et al. Exercise can prevent and reverse the severity of hypertrophic cardiomyopathy. Circ Res. 2006;98(4):540-548.

62. Downing J, Balady GJ. The role of exercise training in heart failure. J Am Coll Cardiol. 2011;58(6):561-569.

63. Takimoto E, Kass DA. Role of oxidative stress in cardiac hypertrophy and remodeling. Hypertension. 2007;49(2):241-248.

64. del Monte F, et al. Restoration of contractile function in isolated cardiomyocytes from failing human hearts by gene transfer of SERCA2a. Circulation. 1999;100(23):2308-2311.

65. Qin F, et al. Hydrogen peroxide-mediated SERCA cysteine 674 oxidation contributes to impaired cardiac myocyte relaxation in senescent mouse heart. J Am Heart Assoc. 2013;2(4):e000184.

66. Duerr GD, et al. Metallothioneins 1 and 2 Modulate Inflammation and Support Remodeling in Ischemic Cardiomyopathy in Mice. Mediators Inflamm. 2016;2016:7174127.

67. Zhang Y, Hu N, Hua Y, Richmond KL, Dong F, Ren J. Cardiac overexpression of metallothionein rescues cold exposure-induced myocardial contractile dysfunction through attenuation of cardiac fibrosis despite cardiomyocyte mechanical anomalies. Free Radic Biol Med. 2012;53(2):194-207.

68. Cai L, et al. Attenuation by metallothionein of early cardiac cell death via suppression of mitochondrial oxidative stress results in a prevention of diabetic cardiomyopathy. J Am Coll Cardiol. 2006;48(8):1688-1697.

69. Song Y, et al. Cardiac metallothionein synthesis in streptozotocin-induced diabetic mice, and its protection against diabetes-induced cardiac injury. Am J Pathol. 2005;167(1):17-26.

70. Cai L, et al. Inhibition of superoxide generation and associated nitrosative damage is involved in metallothionein prevention of diabetic cardiomyopathy. Diabetes. 2005;54(6):1829-1837.

71. Walsh K, Shiojima I. Cardiac growth and angiogenesis coordinated by intertissue interactions. J Clin Invest. 2007;117(11):3176-3179

72. Bernardo BC, McMullen JR. Molecular Aspects of Exercise-induced Cardiac Remodeling. Cardiol Clin. 2016;34(4):515-530

73. Shiojima I, et al. Disruption of coordinated cardiac hypertrophy and angiogenesis contributes to the transition to heart failure. J Clin Invest. 2005;115(8):2108-2118.

74. Sano M, et al. p53-induced inhibition of Hif-1 causes cardiac dysfunction during pressure overload. Nature. 2007;446(7134):444-448.

75. Konhilas JP, et al. Diet and sex modify exercise and cardiac adaptation in the mouse. Am J Physiol Heart Circ Physiol. 2015;308(2):H135-H145.

76. Matsumoto T, Turesson I, Book M, Gerwins P, Claesson-Welsh L. p38 MAP kinase negatively regulates endothelial cell survival, proliferation, and differentiation in FGF-2-stimulated angiogenesis. J Cell Biol. 2002;156(1):149-160.

77. Braz JC, et al. Targeted inhibition of p38 MAPK promotes hypertrophic cardiomyopathy through upregulation of calcineurin-NFAT signaling. J Clin Invest. 2003;111(10):1475-1486.

78. Konhilas JP, Maass AH, Luckey SW, Stauffer BL, Olson EN, Leinwand LA. Sex modifies exercise and cardiac adaptation in mice. Am J Physiol Heart Circ Physiol. 2004;287(6):H2768-H2776.

79. O'Connor PM, Gutterman DD. Resurrecting hope for antioxidant treatment of cardiovascular disease: focus on mitochondria Circ Res. 2010;107(1):9-11

80. Schmidt HH, et al. Antioxidants in Translational Medicine. Antioxid Redox Signal. 2015;23(14):1130-1143 